\title{
BOUNDARY PROBLEMS FOR DIRAC TYPE OPERATORS ON MANIFOLDS WITH MULTI-CYLINDRICAL END BOUNDARIES
}

\author{
PAUL LOYA AND JINSUNG PARK
}

\begin{abstract}
The goal of this paper is to establish a geometric program to study elliptic pseudodifferential boundary problems which arise naturally under cutting and pasting of geometric and spectral invariants of Dirac type operators on manifolds with corners endowed with multi-cylindrical, or $b$-type, metrics and ' $b$-admissible' partitioning hypersurfaces. We show that the Cauchy data space of a Dirac operator on such a manifold is Lagrangian for the self-adjoint case, the corresponding Calderón projector is a $b$-pseudodifferential operator of order 0, characterize Fredholmness, prove relative index formulæ, and solve the Bojarski conjecture.
\end{abstract}

\section{INTRODUCTION}

The purpose of this paper is to establish the geometric theory of elliptic pseudodifferential boundary problems for Dirac type operators on manifolds with multicylindrical end boundaries. The main impetus for this theory is to develop Fredholm and spectral theory and derive gluing formulas for the index, eta invariant, and $\zeta$-determinant of Dirac type operators on such manifolds. In this paper, we focus on the Fredholm theory. We show that the Cauchy data space of such a Dirac operator is Lagrangian for the self-adjoint case, the corresponding Calderón projector is a $b$-pseudodifferential operator of order 0 , and we prove relative index formulæ and solve the Bojarski conjecture for such manifolds. In the forthcoming articles [20], [21] we study the relative and gluing formulæ for the spectral invariants, respectively, for such manifolds.

We begin by describing geometrically our class of manifolds. An $n$-dimensional compact manifold with corners $X$ is a compact topological space locally modelled on $[0, \infty)_{x}^{k} \times \mathbb{R}_{y}^{n-k}$, where $k$ can run between 0 and $n$, such that $X$ has only finitely many boundary hypersurfaces, say $\left\{H_{0}, H_{1}, \ldots, H_{r}\right\}$, where each $H_{i}$ is imbedded in the sense that near the hypersurface $H_{i}$, we have

$$
X \cong[0,1]_{x_{i}} \times H_{i}, \quad i=1,2, \ldots, r \quad, \quad H_{i}=\left\{x_{i}=0\right\} .
$$

We make the assumption that $X$ is connected and $H_{0}$ is an admissible boundary hypersurface (this can be disconnected), which simply means that $H_{0} \cap H_{i} \neq \varnothing$ for $i=1, \ldots, r$; see Figure 1 for examples of manifolds with corners and choices of $H_{0}$. For future convenience in establishing gluing formulas we assume that near $H_{0}$,

$$
X \cong[-1,0]_{u} \times H_{0} \quad, \quad H_{0}=\{u=0\} .
$$

Date: September 29, 2005. file name: LoyaParkCald.tex.

Key words and phrases. Dirac operators; $b$-calculus; manifolds with corners.

2000 Mathematics Subject Classification. Primary: 58J28, 58J52. 

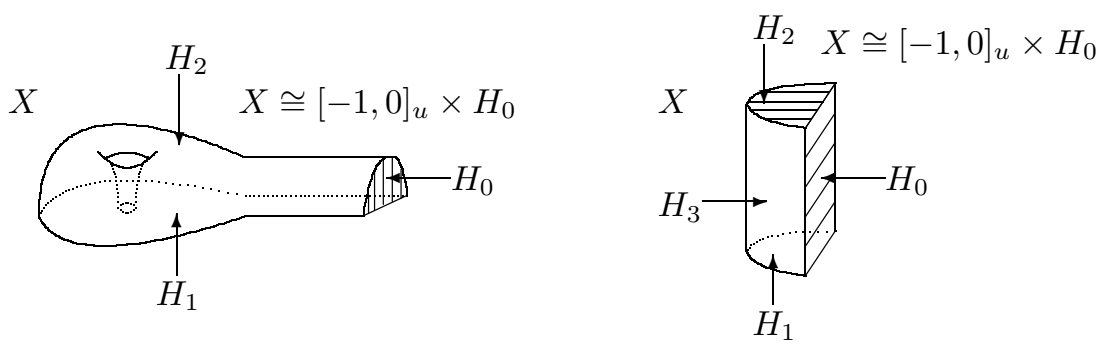

FIgURE 1. Two examples of three-dimensional manifolds with corners (each figure is solid). On the left, all the hypersurfaces are admissible, but we choose $H_{0}$ to be the right 'cap'. On the right, $H_{0}$ and $H_{3}$ are admissible, but $H_{1}$ and $H_{2}$ are not.

How do these manifolds arise? They arise very naturally. One example is to take a (solid) soda can: the round portion is admissible and the top and bottom are not admissible. A related example is to take the soda can and stand it up, then slice it from top to bottom; each piece is a manifold with corners and the newly formed flat side is admissible as seen in the right picture in Figure 1 where we only show the left piece of the cut soda can.

We now put an exact $b$-metric $g$ on $X$ that geometrically pushes each hypersurface $H_{i}, i=1, \ldots, r$, out to infinity and which is smooth up to $H_{0}$; thus the ' $b$-' refers only with respect to $H_{1}, \ldots, H_{r}$. This means that the metric $g$ is smooth up to $H_{0}$ and the metric degenerates up to each $H_{i}, i=1, \ldots, r$, as follows:

$$
g=\sum_{i=1}^{r}\left(\frac{d x_{i}}{x_{i}}\right)^{2}+h
$$

where $h$ is a smooth symmetric two-form on $X$. Explicitly, pick a point $p \in X$, let us say $p \in H_{0} \cap H_{1} \cap \cdots \cap H_{k}$, and $p$ is in no other hypersurfaces; then we assume that there is a common decomposition stemming from (1.1) and (1.2) such that near the point $p$ we can write

$$
X \cong[-1,0]_{u} \times[0,1]_{x}^{k} \times Y,
$$

where $Y$ is the component of $H_{0} \cap H_{1} \cap \cdots \cap H_{k}$ containing $p$. Then in this coordinate patch, $g$ can be written as

$$
g=a(u, x, y) d u^{2}+\left(\frac{d x_{1}}{x_{1}}\right)^{2}+\cdots+\left(\frac{d x_{k}}{x_{k}}\right)^{2}+h(u, x, y),
$$

where $a$ is a smooth positive function (even smooth and positive when $u$ or any $x_{i}$ is zero) and $h$ is a symmetric two-form on $X$ that is smooth near $p$. There is a similar description of $g$ in other patches away from $H_{0}$.

This is the 'compact viewpoint'; there is a 'noncompact viewpoint' in terms of attaching cylinders that might be easier to understand. Let us make the change of variables $t_{i}=\log x_{i}$. Observe that when $x_{i}=1, t_{i}=0$ and as $x_{i} \rightarrow 0, t_{i} \rightarrow-\infty$. Therefore, under this change of variables, the coordinates (1.4) and the metric (1.5) near the point $p$ take the 'multi-cylindrical' forms:

$$
X \cong[-1,0]_{u} \times(-\infty, 0]_{t}^{k} \times Y,
$$


where over this patch,

$$
g=a^{\prime}(u, t, y) d u^{2}+d t_{1}^{2}+\cdots+d t_{k}^{2}+h^{\prime}(u, t, y) .
$$

Here, $a^{\prime}(u, t, y)=a\left(u, e^{t}, y\right)$ and $h^{\prime}(u, t, y)=h\left(u, e^{t}, y\right)$ where we define $e^{t}=$ $\left(e^{t_{1}}, \ldots, e^{t_{k}}\right)$. One can work in this 'noncompact viewpoint' but we choose to work under the compactified viewpoint because from this perspective we have Melrose's $b$-calculus machine [23] at our disposal.

We emphasize that $b$-metrics of the sort (1.3) arise very naturally under cutting: Take a solid soda can as we mentioned before and put an exact $b$-metric on it that geometrically pushes all its hypersurfaces to infinity. Now cut the can from top to bottom as before to get a piece like in the right picture in Figure 1. The resulting metric $g$ is smooth up to $H_{0}$ but is still an exact $b$-metric up to $H_{1}, H_{2}, H_{3}$. Thus, metrics of the sort (1.3) arise naturally in the context of cutting and pasting of geometric and spectral invariants of Dirac type operators on noncompact 'multicylindrical end' manifolds and noncompact partitioning hypersurfaces. We also remark that instead of considering metrics of the form (1.3) with $d x_{i} / x_{i}$ 's, we can consider metrics with $d x_{i} / x_{i}^{2}$ 's. The resulting metric is called an exact cusp metric and very analogous results in this paper hold for such a metric. We choose to work with $b$-metrics only because in the sequel [21] to this paper we shall use certain analytic objects (the $b$-zeta determinant for instance) which are a little more natural to use in the $b$-setting.

Let $E, F$ be Hermitian vector bundles over $X$ and let $\mathcal{D}: C^{\infty}(X, E) \rightarrow C^{\infty}(X, F)$ be a Dirac type operator; that is, a Dirac type operator derived from the metric (1.3) (see Section 2 for a precise definition or Melrose's anticipated [24]). We also assume that on the collar $[-1,0]_{u} \times H_{0}$ of the admissible boundary hypersurface $H_{0}$ with $H_{0}=\{u=0\}$, the vector bundles $E$ and $F$ are isometric to $E_{0}:=\left.E\right|_{H_{0}}$ and $F_{0}:=\left.F\right|_{H_{0}}$, and

$$
\mathcal{D}=G_{u}\left(\partial_{u}+D_{u}\right)
$$

where $G_{u}: E_{0} \rightarrow F_{0}$ is unitary and $D_{u}: C^{\infty}\left(H_{0}, E_{0}\right) \rightarrow C^{\infty}\left(H_{0}, E_{0}\right)$ is a Dirac type operator, and where both $G_{u}$ and $D_{u}$ are smooth up to $u=0$ and restricting there to define a unitary map $G_{0}: E_{0} \rightarrow F_{0}$ and a formally self-adjoint Dirac type operator $D_{0}: C^{\infty}\left(H_{0}, E_{0}\right) \rightarrow C^{\infty}\left(H_{0}, E_{0}\right)$.

We remark that boundary value problems on manifolds with cylindrical end boundaries, which are special cases of manifolds with multi-cylindrical ends boundaries, have been considered by Schrohe [28] and Schrohe and Schulze [29] in the context of Boutet de Monvel's algebra [6] and by Mitrea and Nistor [27] generalizing the method of layer potentials to such manifolds. Grubb [10] (cf. also Grubb and Kokholm [11]) has studied boundary value problems for a class of noncompact manifolds that are Euclidean at $\infty$.

We now state our main results. Given any real $s$, we define $H_{b}^{s}(X, E)$ as the natural $b$-Sobolev space and we define $H_{b}^{\infty}(X, E)$ as the intersection of all $H_{b}^{s}(X, E)$ and we denote by $\widetilde{\Psi}_{b}^{s}\left(H_{0}, E_{0}\right)$ the space of $b$-pseudodifferential operators of order $s$ in the 'calculus with bounds' (see Section 2); similar remarks hold for $F$. The Cauchy data space of $\mathcal{D}$ is by definition

$$
\mathcal{H}(\mathcal{D}):=\left.\operatorname{ker} \mathcal{D}\right|_{H_{0}}=\left\{\left.\phi\right|_{H_{0}} \mid \phi \in H_{b}^{\infty}(X, E), \mathcal{D} \phi=0\right\} \subset H_{b}^{\infty}\left(H_{0}, E_{0}\right),
$$

where ker $\mathcal{D}$ denotes the kernel of $\mathcal{D}$ on $H_{b}^{\infty}(X, E)$. For our first result, we extend the theory and application of the (orthogonalized) Calderón projector to our 
category of manifolds. Here, the Calderón projector was introduced by Calderón [7] and Seeley [30], [31] (cf. also Hörmander [12] and Grubb [8]). The theory and application of the Calderón projector was extended to a class of manifolds which are Euclidean at $\infty$ by Grubb [10] (cf. also Grubb and Kokholm [11]).

Theorem 1.1. The operator

$$
\mathcal{D}: H_{b}^{\infty}(X, E) \rightarrow H_{b}^{\infty}(X, F)
$$

is surjective, restriction to the boundary gives a canonical isomorphism between ker $\mathcal{D}$ and $\mathcal{H}(\mathcal{D})$, and there exists a (unique) orthogonalized Calderón projector $\mathcal{C} \in \widetilde{\Psi}_{b}^{0}\left(H_{0}, E_{0}\right)$ whose image on $H_{b}^{\infty}\left(H_{0}, E_{0}\right)$ is exactly $\mathcal{H}(\mathcal{D})$. Moreover, the $b$ principal symbol of $\mathcal{C}$ is the orthogonal projection onto the eigenspaces of the negative eigenvalues of the corresponding b-principal symbol of $D_{0}$.

We remark that $\mathcal{D}$ is surjective for any real $s$ and not just $s=\infty$. We also remark that we make no invertibility assumptions whatsoever on the Dirac type operator $\mathcal{D}$. This may sound striking because in the case of a manifold with corners with an exact $b$-metric (which pushes all its boundary hypersurfaces to $\infty$ ), it is well-known [19, Cor. 2.5] that a Dirac type operator is Fredholm if and only if all the induced Dirac type operators on the boundary hypersurfaces are invertible (see also Theorem 2.5 and Remark 6.3). Theorem 1.1 shows that with no invertibility assumptions whatsoever, if we cut the manifold forming an admissible face, then the Calderón projector is well-defined at that face, has the same properties as on a compact manifold, and even has the 'nice' structure of a $b$-operator. See Section 3 for an example where we compute the Calderón projector in a model case where none of the induced Dirac operators are invertible. This example also shows that, in general, the Calderón projector $\mathcal{C}$ is not in the 'small' $b$-calculus.

Assume for the moment that $E=F$ and $\mathcal{D}$ is formally self-adjoint. Then in particular, $G_{0}^{2}=-$ Id. One can check that

$$
\Omega(v, w):=\left\langle G_{0} \varphi, \psi\right\rangle_{0} \quad \text { for } \quad \varphi, \psi \in L_{b}^{2}\left(H_{0}, E_{0}\right)
$$

is a Hermitian symplectic form on $L_{b}^{2}\left(H_{0}, E_{0}\right)$ where $\langle,\rangle_{0}$ is the $L_{b}^{2}$ inner product on $H_{0}$. Our second result is

Theorem 1.2. Assume $E=F$ and $\mathcal{D}$ is formally self-adjoint. Then the closure of the Cauchy data space $\mathcal{H}(\mathcal{D})$ in $L_{b}^{2}\left(H_{0}, E_{0}\right)$ is Lagrangian with respect to $\Omega$. In particular, with respect to the decomposition $L_{b}^{2}\left(H_{0}, E_{0}\right)=L_{b}^{2}\left(H_{0}, E_{0}^{+}\right) \oplus L_{b}^{2}\left(H_{0}, E_{0}^{-}\right)$, where $E_{0}^{ \pm}$are the $( \pm i)$-eigenspaces of $G_{0}$, the orthogonalized Calderón projector $\mathcal{C}$ takes the form

$$
\mathcal{C}=\frac{1}{2}\left(\begin{array}{cc}
\mathrm{Id} & \kappa_{0}^{-1} \\
\kappa_{0} & \mathrm{Id}
\end{array}\right),
$$

where $\kappa_{0}: L_{b}^{2}\left(H_{0}, E_{0}^{+}\right) \rightarrow L_{b}^{2}\left(H_{0}, E_{0}^{-}\right)$is a unitary operator.

We remark that Theorem 1.2 - the Lagrangian property of $\mathcal{H}(\mathcal{D})$ - holds without any product structures near $H_{0}$. This is one of the important geometric properties of $\mathcal{H}(\mathcal{D})$, which plays the crucial rôle in the study of the spectral invariants for manifolds with multi-cylindrical end boundaries [20, 21]. In our next theorem, under the condition that $\mathcal{D}$ is of product type near $H_{0}$; that is, in the decomposition (1.6) of $\mathcal{D}$ near $H_{0}$, both $G_{u}$ and $D_{u}$ are constant in $u$, we show that the orthogonalized Calderón projector $\mathcal{C}$ is equal to the Calderón projector defined 
from the Poisson operator of the invertible double of $\mathcal{D}$. Here, the invertible double is defined in Section 4 following the work of Wojciechowski [32].

Theorem 1.3. Assume $E=F$ and $\mathcal{D}$ is formally self-adjoint and assume that $\mathcal{D}$ is of product-type near $H_{0}$. Then the Calderón projector defined by the invertible double of $\mathcal{D}$ equals the orthogonalized projector $\mathcal{C}$.

Back to the general case, using the Calderón projector $\mathcal{C}$, we can characterize the Fredholm properties of $\mathcal{D}$ with other projectors defining the boundary condition. Given an arbitrary projector $\mathcal{P}$ on $L_{b}^{2}\left(H_{0}, E_{0}\right)$, we define

$$
\mathcal{D}_{\mathcal{P}}: \operatorname{dom}\left(\mathcal{D}_{\mathcal{P}}\right) \rightarrow L_{b}^{2}(X, F)
$$

where

$$
\operatorname{dom}\left(\mathcal{D}_{\mathcal{P}}\right):=\left\{\phi \in H_{b}^{1}(X, E) \mid \mathcal{P}\left(\left.\phi\right|_{H_{0}}\right)=0\right\}
$$

Because this domain involves only $\operatorname{ker} \mathcal{P}=\operatorname{ran}(\operatorname{Id}-\mathcal{P})$ we can obtain the same domain by replacing the projector Id $-\mathcal{P}$ with its orthogonalization (cf. Remark 3.5 in [10]). For this reason, we consider only orthogonal projections and we define the smooth self-adjoint Grassmanian $\operatorname{Gr}_{\infty}^{*}(\mathcal{D})$ as those orthogonal projections $\mathcal{P} \in$ $\widetilde{\Psi}_{b}^{0}\left(H_{0}, E_{0}\right)$ such that $\mathcal{P}-\mathcal{C} \in \widetilde{\Psi}^{-\infty}\left(H_{0}, E_{0}\right)$, the space of Green operators (in the calculus with bounds - see the definition (2.12) in Section 2). This implies, in particular, that $\mathcal{P C}: \operatorname{ran} \mathcal{C} \rightarrow \operatorname{ran} \mathcal{P}$ is Fredholm. The following theorem is the multi-cylindrical end version of reduction to the boundary.

Theorem 1.4. For an arbitrary projection $\mathcal{P} \in G r_{\infty}^{*}(\mathcal{D})$, the operator

$$
\mathcal{D}_{\mathcal{P}}: \operatorname{dom}\left(\mathcal{D}_{\mathcal{P}}\right) \rightarrow L_{b}^{2}(X, F)
$$

is Fredholm, and

$$
\operatorname{ind} \mathcal{D}_{\mathcal{P}}=\operatorname{ind}(\mathcal{P}, \mathcal{C})
$$

where $\operatorname{ind}(\mathcal{P}, \mathcal{C}):=\operatorname{ind}(\mathcal{P C}: \operatorname{ran} \mathcal{C} \rightarrow \operatorname{ran} \mathcal{P})$. In particular, for any $\mathcal{P} \in G r_{\infty}^{*}(\mathcal{D})$, the essential spectrum of the operator $\mathcal{D}_{\mathcal{P}}$ in (1.7) has a gap near 0 and for any two projections $\mathcal{P}_{1}, \mathcal{P}_{2} \in G r_{\infty}^{*}(\mathcal{D})$, we have

$$
\text { ind } \mathcal{D}_{\mathcal{P}_{1}}-\operatorname{ind} \mathcal{D}_{\mathcal{P}_{2}}=\operatorname{ind}\left(\mathcal{P}_{1}, \mathcal{P}_{2}\right) \text {. }
$$

As before, we remark that we make no invertibility assumptions whatsoever on $\mathcal{D}$. Again, at first sight, this theorem is quite unbelievable because of the strong invertibility assumptions needed for the boundary Dirac operators in order that the corresponding statements hold for Dirac operators on manifolds with corners with exact $b$-metrics (which push all their boundary hypersurfaces to $\infty$ ). Therefore, the results stated in Theorem 1.4 show the importance of the Calderón projector for the noncompact set-up. Moreover, the model case presented in Section 3 shows the failure of the APS spectral projector to be a pseudodifferential operator. This means that analytically it would be unfeasible to approach boundary problems via the $A P S$ spectral projector and thus illuminates the effectiveness of the Calderón method.

We now describe the Bojarski conjecture. Let $M$ be a smooth manifold with corners with an exact $b$-metric and let $\mathcal{D}: C^{\infty}(M, E) \rightarrow C^{\infty}(M, F)$ be a Dirac type operator. Suppose that $Y$ is a hypersurface inside $M$ that divides $M$ into two manifolds with corners

$$
M=M_{-} \cup M_{+},
$$


where both $M_{ \pm}$are of the form considered in Figure 1 such that $\left.g\right|_{M_{ \pm}}$and $\mathcal{D}_{ \pm}:=$ $\left.\mathcal{D}\right|_{M_{ \pm}}$satisfy the conditions we have already discussed with respect to $Y$, which we assume is admissible for both $M_{ \pm}$. The Bojarski Conjecture [4], later proved by Booß-Bavnbek and Wojciechowski [5, Th. 24.1] for compact smooth manifolds, gives a gluing formula for the index on $M$ in terms of the Fredholm index of pairs of boundary conditions from $M_{ \pm}$. Here is the multi-cylindrical end version.

Theorem 1.5. Suppose that $\mathcal{D}: H_{b}^{1}(M, E) \rightarrow L_{b}^{2}(M, F)$ is Fredholm. Then for arbitrary projections $\mathcal{P}_{ \pm} \in G r_{\infty}^{*}\left(\mathcal{D}_{ \pm}\right)$, we have

$$
\operatorname{ind} \mathcal{D}=\operatorname{ind} \mathcal{D}_{\mathcal{P}_{-}}+\operatorname{ind} \mathcal{D}_{\mathcal{P}_{+}}-\operatorname{ind}\left(\mathcal{P}_{-}, \mathrm{Id}-\mathcal{P}_{+}\right) \text {. }
$$

In Section 2, we give a self-contained, introductory presentation of $b$-pseudodifferential operators on manifolds with corners. In Section 3 we compute the Calderón projector explicitly in the 'model' case when $X=[0,1]_{u} \times[0, \infty)^{\ell}$. We prove that the Calderón projector exists and is in the $b$-calculus with bounds and we compute its $b$-principal symbol. In Section 4 , we construct the invertible double of a Dirac type operator over a manifold with multi-cylindrical end. In Sections 5 and 6 , we prove Theorems 1.1, 1.2, 1.3, 1.4 and 1.5 using the results proved in the previous sections.

\section{INTRODUCTION TO $b$-OPERATORS ON MANIFOLDS WITH CORNERS}

We review the $b$-calculus on manifolds with corners. For more on these topics, see Melrose [23], Mazzeo [22], or the appendices of Melrose and Piazza [26], and Melrose and Nistor [25].

2.1. $b$-pseudodifferential operators. Let $M$ be a compact manifold with corners with local expressions of the form (1.1) endowed with an exact $b$-metric of the form (1.3) (where exact means there are no admissible boundary hypersurfaces; all the boundary hypersurfaces are geometrically at $\infty$ ). In particular, near any point $p \in M$, as explained around (1.4) we can write

$$
M \cong[0,1]_{v}^{\kappa} \times Y,
$$

where the $v_{i}$ 's represent those boundary defining functions $x_{j}$ that vanish at $p$ and $Y$ is the component containing $p$ where all the $v_{i}$ 's vanish. A codimension $\kappa$ face, $\kappa \geq 1$, of $M$ is a nonempty connected component of the intersection of $\kappa$ hypersurfaces of $M$. In particular, a boundary hypersurface is just a codimension one face of $M$. The largest $\kappa$ such that $M$ has a (nonempty) condimension $\kappa$ face is called the codimension of $M$.

We now describe the 'small' calculus. Let $\dot{C}^{\infty}(M)$ denote the space of smooth functions on $M$ that vanish to infinite order at the boundary of $M$; that is, in Taylor series at any $x_{i}$ in a local patch such as (2.1). We first define the $b$-smoothing operators $\Psi_{b}^{-\infty}(M)$ (which are not genuine smoothing operators). These are operators $R$ on $\dot{C}^{\infty}(M)$ described in local coordinates as follows. Let $\mathscr{U}$ and $\mathscr{U}^{\prime}$ be coordinate patches on $M$ of the form (2.1) where we take coordinates on the corresponding $Y$ 's for $\mathscr{U}$ and $\mathscr{U}^{\prime}$. We allow $\kappa=0$ in (2.1) for either $\mathscr{U}$ or $\mathscr{U}^{\prime}$, which means that the coordinate patch is located in the interior of our manifold $M$. Let $v=\left(v_{1}, \ldots, v_{\ell}\right)$ denote those boundary defining functions, if any, that are common to both coordinate patches $\mathscr{U}$ and $\mathscr{U}^{\prime}$, so that

$$
\mathscr{U}=[0,1]_{v}^{\ell} \times \mathscr{V} \quad, \quad \mathscr{U}^{\prime}=[0,1]_{v}^{\ell} \times \mathscr{V}^{\prime}
$$


where $\mathscr{V} \subset[0, \infty)^{k} \times \mathbb{R}^{n-\ell-k}$ and $\mathscr{V}^{\prime} \subset[0, \infty)^{k^{\prime}} \times \mathbb{R}^{n-\ell-k^{\prime}}$. Let $y$ denote the coordinates on $\mathscr{V}$ and $y^{\prime}$ the coordinates on $\mathscr{V}^{\prime}$. Then given any open set $\mathscr{W}$ with compact closure in $\mathscr{U}^{\prime}$, for any $\phi \in \dot{C}^{\infty}(M)$ having support in $\mathscr{W}$, the restriction of $R \phi$ to $\mathscr{U}$ is of the form

$$
R \phi=\int_{\mathscr{U}^{\prime}} R\left(v, \frac{v}{v^{\prime}}, y, y^{\prime}\right) \phi\left(v^{\prime}, y^{\prime}\right) d g\left(v^{\prime}, y^{\prime}\right),
$$

where $v / v^{\prime}:=\left(v_{1} / v_{1}^{\prime}, \ldots, v_{\ell} / v_{\ell}^{\prime}\right)$ and where $R\left(v, z, y, y^{\prime}\right)$ has the following regularity properties: It is smooth in all variables, vanishes to infinite order with all derivatives at any $z_{i}=0$ or as $z_{i} \rightarrow \infty$, and vanishes to infinite order at any $y_{i}=0$ or $y_{i}^{\prime}=0$ when the corresponding zero set represents a boundary hypersurface of $M$.

We now consider the general case of an operator $A \in \Psi_{b}^{m}(M), m \in \mathbb{R}$, which is an operator on $\dot{C}^{\infty}(M)$ described in local coordinates as follows. Let $\mathscr{U}$ and $\mathscr{U}^{\prime}$ be coordinate patches of the form $(2.2)$ and let $\mathscr{W}$ be an open set with compact closure in $\mathscr{U}^{\prime}$.

(I) If $\mathscr{U}$ and $\mathscr{U}^{\prime}$ are disjoint, then given any $\phi \in \dot{C}^{\infty}(M)$ having support in $\mathscr{W}$, the restriction of $A \phi$ to $\mathscr{U}$ is given by an operator $R \in \Psi_{b}^{-\infty}(M)$ as in (2.3).

(II) Suppose now that $\mathscr{U}=\mathscr{U}^{\prime}=[0,1]_{v}^{\ell} \times \mathbb{R}_{y}^{n-\ell}$. Then there is a function $a(v, y, \xi)$, smooth in $(v, y) \in \mathscr{U}$ and a classical symbol of order $m$ in $\xi$, such that given any $\phi \in \dot{C}^{\infty}(M)$ having support in $\mathscr{W}$, we have

$$
A \phi=\int_{\mathbb{R}^{n}} v^{i \tau} e^{i y \cdot \eta} a(v, y, \tau, \eta) \hat{\phi}(\xi) d \tau d \eta,
$$

where $\xi=(\tau, \eta), v^{i \tau}=v_{1}^{i \tau_{1}} \cdots v_{\ell}^{i \tau_{\ell}}$, and $\hat{\phi}(\xi)$ is the Mellin transform in $v$ and the Fourier transform in $y$ of $\phi$,

$$
\hat{\phi}(\tau, \eta)=\int_{\mathscr{U}} v^{-i \tau} e^{-i y \cdot \eta} \phi(v, y) \frac{d v}{v} d y .
$$

We also assume that $a(v, y, \xi)$ with all its derivatives extends to be an entire function of $\tau$, and for $|\operatorname{Im} \tau|$ bounded by any fixed number, is a classical symbol of order $m$ in $(\tau, \eta)$ as $|\operatorname{Re} \tau|,|\eta| \rightarrow \infty$; this is a "lacunary" type condition for $\tau \in \mathbb{C}^{k}$ as described by Hörmander [13, p. 114].

The space $\Psi_{b}^{m}(M)$ is called the small calculus of b-pseudodifferential operators of order $m$. It also turns out that any $A \in \Psi_{b}^{m}(M)$ defines a continuous map on $C^{\infty}(M)$. In the literature, $b$-operators are most often presented in terms of their Schwartz kernels. Observe that combining (2.4) and (2.5), we see that the Schwartz kernel of $A$ on the product $M \times M$ near the diagonal is of the form

$$
K_{A}=\int_{\mathbb{R}^{n}}\left(\frac{v}{v^{\prime}}\right)^{i \tau} e^{i\left(y-y^{\prime}\right) \cdot \eta} a(v, y, \tau, \eta) d \tau d \eta \cdot \frac{d v^{\prime}}{v^{\prime}} d y^{\prime},
$$

where $(v, y)$ are coordinates on the left factor of $M$ and $\left(v^{\prime}, y^{\prime}\right)$ are the same coordinates on the right factor of $M$. Introducing 'logarithmic coordinates' $w=$ $\left(\log v_{1}, \ldots, \log v_{\ell}, y\right)$, we can write this kernel as

$$
K_{A}=\int_{\mathbb{R}^{n}} e^{i\left(w-w^{\prime}\right) \cdot \xi} a(v, y, \xi) d \xi \cdot d w^{\prime},
$$

which looks like the Schwartz kernel of a pseudodifferential operator that we are used to. However, the 'singular' presentation (2.6) has certain advantages; perhaps the major one is that it, quite remarkably, actually simplifies the proofs of the composition and mapping properties of these operators. 
The space of $b$-pseudodifferential operators has many of the same properties as the common ones on compact boundaryless manifolds. For example, $\operatorname{Diff}_{b}^{m}(M) \subset$ $\Psi_{b}^{m}(M)$, where $\operatorname{Diff}_{b}^{m}(M)$ is the space of totally characteristic differential operators, which are operators taking the following form over a patch $[0,1]_{v}^{\ell} \times \mathbb{R}_{y}^{n-\ell}$ :

$$
P=\sum_{|\alpha|+|\beta| \leq m} a_{\alpha, \beta}(v, y)\left(v \partial_{v}\right)^{\alpha} \partial_{y}^{\beta},
$$

where the $a_{\alpha, \beta}$ 's are smooth in $(v, y) \in[0,1]_{v}^{\ell} \times \mathbb{R}_{y}^{n-\ell}$. The space of $\Psi_{b}^{\bullet}(M)$ is also a symbolically filtered $*$-algebra of operators. Thus, it is closed under taking adjoints and compositions, and there is a ' $b$-' principal symbol map preserving these operations obtained by taking the leading homogeneous term of each symbol $a(v, y, \xi)$ in the local representation (2.7). For example, the $b$-principal symbol of $P$ in $(2.8)$ is

$$
{ }^{b} \sigma_{m}(P)(v, y, \xi)=\sum_{|\alpha|+|\beta|=m} a_{\alpha, \beta}(v, y)(i \tau)^{\alpha}(i \eta)^{\beta}, \xi=(\tau, \eta) .
$$

The $b$-principal symbol turns out to be a function on the $b$-cotangent bundle ${ }^{b} T^{*} M$ of $M$ minus the zero section [23, p. 30]. An operator $A$ is said to be $b$-elliptic if its $b$-principal symbol is invertible. The space $L_{b}^{2}(M)$ consists of those functions on $M$ that are square integrable with respect to $d g$ and for any $m \in \mathbb{R}$, the Sobolev space $H_{b}^{m}(M)$ consists of those distributions $\phi$ on $M$ having the property that $A \phi \in$ $L_{b}^{2}(M)$ for all $A \in \Psi_{b}^{m}(M)$; one can check that $H_{b}^{m}(M)$ is just the usual Sobolev space in the interior of $M$ and the Mellin transform based Sobolev space in the normal variables to the hypersurfaces of $M$. We define $H_{b}^{\infty}(M)=\bigcap_{m \in \mathbb{R}} H_{b}^{m}(M)$. Then any $A \in \Psi_{b}^{m}(M)$ defines a continuous linear map

$$
A: H_{b}^{s}(M) \rightarrow H_{b}^{s-m}(M) \quad, \quad s \in \mathbb{R} \cup\{\infty\} .
$$

Unfortunately, the 'small' space $\Psi_{b}^{\bullet}(M)$ is not spectrally invariant in the sense that this set is not closed under inversion, when inverses exist; however, inverses can be found in the 'larger' calculus with bounds, which we now describe. Let $\theta>0$. We define $\Psi_{b}^{m, \theta}(M)$ as those operators $A$ that satisfy (I) and (II) as before, but with the following modifications: In (I), we can write $R$ as in (2.3):

$$
R \phi=\int_{\mathscr{U}^{\prime}} R\left(v, \frac{v}{v^{\prime}}, y, y^{\prime}\right) \phi\left(v^{\prime}, y^{\prime}\right) d g\left(v^{\prime}, y^{\prime}\right),
$$

but now $R\left(v, z, y, y^{\prime}\right)$ has the following 'boundedness' properties: For each $i=$ $1, \ldots, \ell$, we require that

$$
R\left(v, z, y, y^{\prime}\right)=R_{1, i}\left(v, z, y, y^{\prime}\right)+v_{i}^{\theta+\varepsilon} R_{2, i}\left(v, z, y, y^{\prime}\right)
$$

where $R_{1, i}, R_{2, i}$ are smooth for $v, y, y^{\prime}$ in the interior of $M$ and in $z>0$, and there is an $\varepsilon>0$ such that given any constant coefficient $b$-differential operators $P$ in the variables $z, y, y^{\prime}$ and $Q$ in the variables $v, z, y, y^{\prime}$, the functions $\partial_{v}^{\alpha} P R_{1, i}$ (for any $\alpha$ ) and $Q R_{2, i}$ are bounded for all $v, z, y, y^{\prime}$, continuous at each $v_{j}=0$, vanishes to order $z_{j}^{\theta+\varepsilon}$ and $z_{j}^{-\theta-\varepsilon}$ at each $z_{j}=0$ and as $z_{j} \rightarrow \infty$, respectively, and vanishes to order $y_{j}^{\theta+\varepsilon}$ at any $y_{j}=0$ and $\left(y_{j}^{\prime}\right)^{\theta+\varepsilon}$ at any $y_{j}^{\prime}=0$ if any of these sets represent boundary hypersurfaces of $M$. In (II), for each $i$, we require that

$$
a(v, y, \xi)=a_{1, i}(v, y, \xi)+v_{i}^{\theta+\varepsilon} a_{2, i}(v, y, \xi),
$$


where $a_{1, i}(v, y, \xi)$ is smooth in all variables and all $b$-derivatives of $a_{2, i}(v, y, \xi)$ are continuous (here ' $b$-' refers only with respect to $v$ ), and $a_{1, i}$ and $a_{2, i}$ satisfy the lacunary condition for $\tau$ in the strip $|\operatorname{Im} \tau| \leq \theta+\varepsilon$.

This definition of the calculus with bounds can be found in [16, p. 88] or [17, p. 1262] from the 'blown-up picture'. When $\theta$ is not important (as it will not be for this paper), we shall use the space

$$
\widetilde{\Psi}_{b}^{m}(M)=\bigcup_{\theta>0} \Psi_{b}^{m, \theta}(M) .
$$

These spaces form the calculus with bounds and they too form an algebra in the sense that $\Psi_{b}^{m, \theta}(M) \circ \Psi_{b}^{m^{\prime}, \theta^{\prime}}(M) \subset \Psi_{b}^{m+m^{\prime}, \theta^{\prime}}(M)$, where $\theta^{\prime}=\min \left\{\theta, \theta^{\prime}\right\}$. They also define continuous maps between $b$-Sobolev spaces.

2.2. The normal operator and Fredholm properties. The normal operator governs the Fredholm $b$-pseudodifferential operators. Let $Y$ be a codimension $\ell$ boundary face of $M$. Assume that $Y$ is a component of $H_{i_{1}} \cap \cdots \cap H_{i_{\ell}}$, where $i_{1}<\cdots<i_{\ell}$, so that $x_{i_{1}}, \ldots, x_{i_{\ell}}$ are defining functions for $M$. Then near $Y$ (cf. (2.1)) we have

$$
M \cong[0,1]_{v}^{\ell} \times Y, \quad v=\left(x_{i_{1}}, \ldots, x_{i_{\ell}}\right) .
$$

Given $A \in \Psi_{b}^{m}(M)$, the normal operator of $A$ at $Y$ is defined as follows. Given a function $\psi \in C^{\infty}(Y)$, let $\phi \in C^{\infty}(M)$ be any smooth function such that $\left.\phi\right|_{Y}=$ $\psi$. The properties of the small calculus imply that given any fixed $\tau \in \mathbb{C}^{\ell}$, the function $v^{-i \tau} A\left(v^{i \tau} \phi\right)$ defines a smooth function on $M$; in particular, this function is continuous at $Y$. Restricting this function to $Y$ defines the normal operator of $A$ at $Y$ :

$$
N_{Y}(A)(\tau) \psi:=\left.\left(v^{-i \tau} A\left(v^{i \tau} \phi\right)\right)\right|_{Y} .
$$

This operator does not depend on the choice of extension $\phi$. Moreover, it readily follows that

$$
N_{Y}\left(A^{*}\right)(\tau)=N_{Y}(A)(\bar{\tau})^{*}, \quad N_{Y}(A B)(\tau)=N_{Y}(A)(\tau) N_{Y}(B)(\tau)
$$

for any $B \in \Psi_{b}^{m^{\prime}}(M)$ and all $\tau \in \mathbb{C}^{\ell}$. For instance, to prove the composition property, let $\phi$ be an extension of $\psi$ as before, and note that

$$
\begin{aligned}
N_{Y}(A B)(\tau) \psi=\left.\left(v^{-i \tau} A B v^{i \tau} \phi\right)\right|_{Y} & =\left.\left(v^{-i \tau} A v^{i \tau}\left(v^{-i \tau} B v^{i \tau} \phi\right)\right)\right|_{Y} \\
& =N_{Y}(A)(\tau)\left(\left.\left(v^{-i \tau} B v^{i \tau} \phi\right)\right|_{Y}\right) \\
& =N_{Y}(A)(\tau)\left(N_{Y}(B)(\tau) \phi\right) .
\end{aligned}
$$

The following theorem characterizes Fredholm $b$-operators in terms of normal operators. The proof can be found in the appendix of [19].

Theorem 2.1. If $A \in \Psi_{b}^{m}(M), m \in \mathbb{R}^{+}$, then given any $s \in \mathbb{R}$ or $s=\infty$, the following are equivalent:

(1) $A: H_{b}^{s}(M) \rightarrow H_{b}^{s-m}(M)$ is Fredholm.

(2) $A$ is b-elliptic and $N_{H}(A)(\tau): H_{b}^{s}(H) \rightarrow H_{b}^{s-m}(H)$ is invertible for all $\tau \in \mathbb{R}$ for each boundary hypersurface $H$.

(3) $A$ is b-elliptic and $N_{Y}(A)(\tau): H_{b}^{s}(Y) \rightarrow H_{b}^{s-m}(Y)$ is invertible for all $\tau \in \mathbb{R}^{\ell}$ for each codimension $\ell$ face $Y$ of $M$, for all $\ell \geq 1$. 
If the statements (1), (2), or (3) hold for one s, then they automatically hold for any other $s$.

Because of this theorem, when we say $A \in \Psi_{b}^{m}(M)$ is Fredholm we can just fix $s=m$ so we can focus strictly on $A: H_{b}^{m}(M) \rightarrow L_{b}^{2}(M)$.

Finally, we remark that elements of $\widetilde{\Psi}_{b}^{-\infty}(M)$ are not compact. The following theorem characterizes compact $b$-operators in terms of normal operators; see $[19$, Appendix] for the proof.

Theorem 2.2. For $A \in \widetilde{\Psi}_{b}^{-\infty}(M)$, the following are equivalent:

(1) $A: L_{b}^{2}(M) \rightarrow L_{b}^{2}(M)$ is compact.

(2) $N_{H}(A)(\tau) \equiv 0$ for each boundary hypersurface $H$.

(3) $N_{Y}(A)(\tau) \equiv 0$ for each codimension $\ell$ face $Y$ of $M$, for all $\ell \geq 1$.

We define $\widetilde{\Psi}^{-\infty}(M)$ - the (weak) Green operators (here we follow Schulze's terminology as explained in [15, Def. 3.6]) - as all elements of $\widetilde{\Psi}_{b}^{-\infty}(M)$ that are compact. These operators can be described explicitly as follows. Let $\rho=x_{1} x_{2} \cdots x_{r}$ be the product of all the boundary defining functions of $M$. Then $\widetilde{\Psi}^{-\infty}(M)$ consists of operators having Schwartz kernels of the form, for some $\theta>0$ :

$$
K \in \widetilde{\Psi}^{-\infty}(M) \Longleftrightarrow K=\rho(p)^{\theta} \rho(q)^{\theta} R(p, q) d g(q)
$$

where for any $b$-differential operators $P_{p}$ and $Q_{q}$ acting on the variables $p$ and $q$, respectively, the function $P_{p} Q_{q} R(p, q)$ is bounded. Then $K$ maps $H_{b}^{s}(M)$ to $\rho^{\theta} H_{b}^{\infty}(M)$ and defines a compact operator on $H_{b}^{s}(M)$ for any $s$. The $b$ subscript in $\widetilde{\Psi}_{b}^{m}(M)$ has to do with special pseudodifferential structure at the boundary of $M$, but operators in $\widetilde{\Psi}^{-\infty}(M)$ vanish at the boundary, which accounts for the 'missing' $b$ subscript for these compact operators.

The next theorem describes the generalized inverse of Fredholm operators.

Theorem 2.3. If $A \in \Psi_{b}^{m}(M), m \in \mathbb{R}^{+}$, is Fredholm, then its generalized inverse, $G: L_{b}^{2}(M) \rightarrow H_{b}^{m}(M)$, is in the full calculus: $G \in \widetilde{\Psi}_{b}^{-m}(M)$. Here, the generalized inverse is defined by the equations

$$
A G=\mathrm{Id}-\Pi_{1} \quad, \quad G A=\mathrm{Id}-\Pi_{0},
$$

where $\Pi_{0}, \Pi_{1} \in \widetilde{\Psi}^{-\infty}(M)$ are the orthogonal projections onto the null space of $A$ in $H_{b}^{m}(M)$ and off the range of $A$ in $L_{b}^{2}(M)$, respectively. Moreover, $\operatorname{ker} A \subset$ $\rho^{\theta} H_{b}^{\infty}(M)$, and coker $A \cong \operatorname{ker} A^{*} \subset \rho^{\theta} H_{b}^{\infty}(M)$ for some $\theta>0$.

In the following theorem, we give another characterization of Fredholmness which is quite useful in practice rather than in theory, and it will be exploited in a moment to prove the subsequent Theorem 2.5 for Dirac operators.

Theorem 2.4. If $A \in \Psi_{b}^{m}(M), m \in \mathbb{R}^{+}$, then given any $s \in \mathbb{R}$ or $s=\infty$, the following are equivalent:

(1) $A: H_{b}^{s}(M) \rightarrow H_{b}^{s-m}(M)$ is a Fredholm.

(2) $A$ is b-elliptic and $N_{Y}(A)(\tau): H_{b}^{s}(Y) \rightarrow H_{b}^{s-m}(Y)$ has kernel (null space) 0 for all $\tau \in \mathbb{R}^{\ell}$ for each codimension $\ell$ face $Y$ of $M$, for all $\ell \geq 1$.

If the statements (1) and (2) hold for one s, then they automatically hold for any other $s$. 
Proof. By Theorem 2.1, it suffices to show that if $A$ is $b$-elliptic and $N_{Y}(A)(\tau)$ : $H_{b}^{s}(Y) \rightarrow H_{b}^{s-m}(Y)$ has kernel 0 for all $\tau \in \mathbb{R}^{\ell}$ for each codimension $\ell$ face $Y$ of $M$, for all $\ell \geq 1$, then $N_{Y}(A)(\tau)$ must be invertible. We first note that being $b$-elliptic, $A$ has a 'small' parametrix $B \in \Psi_{b}^{-m}(M)$ such that $A B=\mathrm{Id}-R_{1}, B A=\mathrm{Id}-R_{2}$ with $R_{j} \in \Psi_{b}^{-\infty}(M)$. Let $Y$ be any boundary face of $M$. Then taking normal operators, we obtain

$$
\begin{gathered}
N_{Y}(A)(\tau) N_{Y}(B)(\tau)=\mathrm{Id}-N_{Y}\left(R_{1}\right)(\tau) \\
N_{Y}(B)(\tau) N_{Y}(A)(\tau)=\mathrm{Id}-N_{Y}\left(R_{2}\right)(\tau) .
\end{gathered}
$$

Since the $R_{j}$ 's are of order $-\infty$, it follows that $N_{Y}(A)(\tau)$ is $b$-elliptic for all $\tau \in \mathbb{R}^{\ell}$ (with $\ell$ the codimension of $Y$ in $M$ ), and as $|\tau| \rightarrow \infty, N_{Y}\left(R_{j}\right)(\tau) \rightarrow 0$ uniformly in the topology of $\Psi_{b}^{-\infty}(Y)$, and hence we can invert each operator on the right in (2.13) on $L_{b}^{2}$ for $|\tau|$ sufficiently large. In conclusion, $N_{Y}(A)(\tau)$ is a $b$-elliptic family that is invertible for $|\tau|$ sufficiently large.

We are now ready to prove our theorem by induction. Let $\ell_{0}$ be the codimension of $M$. Consider the case when $Y$ is a codimension $\ell_{0}$ face of $M$, in which case $Y$ is a compact boundaryless manifold. Then as we showed above, $N_{Y}(A)(\tau)$ is a family of elliptic (and hence Fredholm) operators on a closed manifold that is invertible for $|\tau|$ sufficiently large. Thus, $N_{Y}(A)(\tau)$ always has index zero. By assumption, $N_{Y}(A)(\tau)$ has kernel 0 , so $N_{Y}(A)(\tau)$ must in fact be invertible. This proves that the claim is true for $\ell_{0}$. Let $\ell_{0} \geq \ell_{k}+1>1$ and assume that all the normal operators of $A$ are invertible at all faces of $M$ with codimension $\geq \ell_{k}+1$. Let $Y$ be a face of $M$ with codimension $\ell_{k}$. Then $Y$ is a manifold with corners whose boundary hypersurfaces are codimension $\ell_{k}+1$ faces of $M$. All its normal operators are invertible by induction hypothesis. Now as we showed above, $N_{Y}(A)(\tau)$ is a family of $b$-elliptic operators on $Y$ that is invertible for $|\tau|$ sufficiently large, therefore by invertibility of its normal operators, $N_{Y}(A)(\tau)$ is a family of Fredholm operators with index zero. By assumption, this family has kernels 0 , so $N_{Y}(A)(\tau)$ must in fact be invertible for all $\tau$. This completes our proof.

Before speaking about Dirac type operators, we note that everything we have said for operators on functions works equally well for operators acting between sections of vector bundles with the obvious modifications.

2.3. Dirac type operators. We now define operators 'of Dirac type'. An operator $\mathcal{D}$ is called a $\left(b\right.$-)Dirac type operator if $\mathcal{D} \in \operatorname{Diff}_{b}^{1}(M, E, F)$, is $b$-elliptic with ${ }^{b} \sigma_{1}(\mathcal{D})^{2}=$ the metric $g$, and near each codimension $\ell$ face $Y$ of $M$, in a decomposition (2.10), $M \cong[0,1]_{v}^{\ell} \times Y$ near $Y$, we can write

$$
\mathcal{D}=G_{1} v_{1} \partial_{v_{1}}+\cdots+G_{\ell} v_{\ell} \partial_{v_{\ell}}+B_{Y}+\mathcal{O}(v),
$$

where $B_{Y} \in \operatorname{Diff}_{b}^{1}\left(Y, E_{Y}, F_{Y}\right)$ with $E_{Y}:=\left.E\right|_{Y}$ and $F_{Y}:=\left.F\right|_{Y}, \mathcal{O}(v)$ is a first order $b$-differential operator that vanishes at $Y$, and where the maps $G_{j}: E_{Y} \rightarrow F_{Y}$ are unitary maps satisfying the relations

$$
G_{j}^{*} G_{k}=-G_{k}^{*} G_{j} \quad(j \neq k) \quad, \quad G_{j}^{*} B_{Y}=B_{Y}^{*} G_{j} .
$$

By definition of normal operator, we have

$$
N_{Y}(\mathcal{D})(\tau)=G_{1} i \tau_{1}+\cdots+G_{\ell} i \tau_{\ell}+B_{Y}
$$

where the $G_{j}$ 's and $B_{Y}$ satisfy (2.15); in fact, one can easily show that (2.16) implies (2.14) so we can define 'of Dirac type' using the normal operator expression 
(2.16) instead of the expression (2.14). The operator $B_{Y}$ is called the induced Dirac operator on $Y$. The word 'induced' follows from the fact that if we set $v_{1}=\cdots=v_{\ell}=0$ in $(2.16)$ we get exactly $B_{Y}$.

Theorem 2.5. A Dirac type operator $\mathcal{D} \in \operatorname{Diff}_{b}^{1}(M, E, F)$ is Fredholm if and only if for each boundary face $Y$ of $M$, the induced Dirac type operator $B_{Y}$ on $Y$ has kernel 0 on $L_{b}^{2}\left(Y, E_{Y}\right)$.

Proof. Let $Y$ be a codimension $\ell$ face of $M$ and fix any $0 \leq j \leq \ell$. We prove that $N_{Y}(\mathcal{D})(\tau)$ is invertible for all $\tau \in \mathbb{R}^{\ell}$ with $\tau_{j} \neq 0$. Indeed, observe that

$$
N_{Y}(\mathcal{D})(\tau)=i G_{1} \tau_{1}+\cdots+i G_{\ell} \tau_{\ell}+B_{Y}=G_{j}\left[i \tau_{j}+A_{j}(\tau)\right],
$$

where

$$
A_{j}(\tau)=\sum_{k \neq j} G_{j}^{*} G_{k} i \tau_{k}+G_{j}^{*} B_{Y}
$$

Since $G_{j}^{*} G_{k}=-G_{k}^{*} G_{j}$ and $G_{j}^{*} B_{Y}=B_{Y}^{*} G_{j}$, the operator $A_{j}(\tau)$ is formally selfadjoint for all $\tau \in \mathbb{R}^{\ell}$. It follows that $N_{Y}(\mathcal{D})(\tau)$ is invertible for all $\tau \in \mathbb{R}^{\ell}$ with $\tau \neq 0$. It has kernel 0 at $\tau=0$ if and only if $B_{Y}$ has kernel 0 . The Fredholm property of $\mathcal{D}$ now follows Theorem 2.4.

Much of what we have studied on $M$ works for our manifold with corners $X$ with an admissible hypersurface $H_{0}$. Take any manifold with corners $M$ with an exact $b$-metric (pushing all its boundary hypersurfaces to $\infty$ ) that contains $X$ as a smooth submanifold; e.g. take the manifold $M=\widetilde{X}$ shown in Figure 3 of Section 4, which is obtained from $X$ by essentially doubling it across $H_{0}$. Then $M$ is a manifold with corners of the type we've been studying, so $H_{b}^{s}(M)$, $\operatorname{Diff}_{b}^{m}(M)$, and $\Psi_{b}^{s}(M)$ are defined. Now simply define $H_{b}^{s}(X), \operatorname{Diff}_{b}^{m}(X)$, and $\Psi_{b}^{s}(X)$ to be the restrictions to $X$ of $H_{b}^{s}(M), \operatorname{Diff}_{b}^{m}(M)$, and $\Psi_{b}^{s}(M)$, respectively. These definitions are determined independently of the choice of extension $M$. We now describe Dirac type operators on $X$.

Let $E, F$ be Hermitian vector bundles over $X$ and let $\mathcal{D}: C^{\infty}(X, E) \rightarrow C^{\infty}(X, F)$ be a Dirac type operator; this means that $\mathcal{D}$ is the restriction to $X$ of a Dirac type operator on $M$. We assume that on the collar $[-1,0]_{u} \times H_{0}$ of the admissible boundary hypersurface $H_{0}$ with $H_{0}=\{u=0\}$, the vector bundles $E$ and $F$ are isometric to $E_{0}:=\left.E\right|_{H_{0}}$ and $F_{0}:=\left.F\right|_{H_{0}}$, and

$$
\mathcal{D}=G_{u}\left(\partial_{u}+D_{u}\right)
$$

where $G_{u}: E_{0} \rightarrow F_{0}$ is unitary and $D_{u} \in \operatorname{Diff}_{b}^{1}\left(H_{0}, E_{0}\right)$ is a Dirac type operator on $H_{0}$, and where both $G_{u}$ and $D_{u}$ are smooth up to $u=0$ and restricting there to define a unitary map $G_{0}: E_{0} \rightarrow F_{0}$ and a formally self-adjoint Dirac type operator $D_{0} \in \operatorname{Diff}_{b}^{1}\left(H_{0}, E_{0}\right)$. Note that $H_{0}$ is an example of an ' $M$ ' as we have been considering in this section. We end this section with Green's formula. Let $\mathcal{D}^{*}$ : $C^{\infty}(X, F) \rightarrow C^{\infty}(X, E)$ denote the formal adjoint of $\mathcal{D}$. Then given $e \in H_{b}^{1}(X, E)$ and $f \in H_{b}^{1}(X, F)$, we have

$$
\langle\mathcal{D} e, f\rangle_{X}-\left\langle e, \mathcal{D}^{*} f\right\rangle_{X}=\left\langle G_{0} e_{0}, f_{0}\right\rangle_{0}
$$

where $e_{0}:=\left.e\right|_{H_{0}}, f_{0}:=\left.f\right|_{H_{0}}$, and $\langle,\rangle_{X}$ and $\langle,\rangle_{0}$ denote $L_{b}^{2}$-inner products over $X$ and $H_{0}$, respectively. The proof of this formula is identical with the proof in the compact case with no changes. 


\section{An illuminating EXAmPle}

In this section we compute the orthogonalized Calderón projector for a 'model' Dirac operator. This example illustrates the basic characteristics of Calderón projectors in the general case. Let $R>0$ and set $X_{R}=[0, R]_{u} \times \overline{\mathbb{R}}_{+}^{\ell}$ where $\overline{\mathbb{R}}_{+}=[0, \infty)$. In this case,

$$
Y_{R}=\partial X_{R}=\left(\{0\} \times \overline{\mathbb{R}}_{+}^{\ell}\right) \sqcup\left(\{R\} \times \overline{\mathbb{R}}_{+}^{\ell}\right) \equiv \overline{\mathbb{R}}_{+}^{\ell} \sqcup \overline{\mathbb{R}}_{+}^{\ell} .
$$

Consider the (b-) Dirac operator

$$
\mathcal{D}=G_{0} \partial_{u}+G_{1} v_{1} \partial_{v_{1}}+\cdots+G_{\ell} v_{\ell} \partial_{v_{\ell}} \in \operatorname{Diff}_{b}^{1}\left(X_{R}, E\right)
$$

where $E$ is a Hermitian Clifford module (that is, we consider the trivial bundle $X_{R} \times E$ over $X_{R}$ ) and the $G_{j}$ 's are unitary matrices on $E$ satisfying

$$
G_{j}^{2}=-\mathrm{Id}, \quad G_{j}^{*}=-G_{j}, \quad G_{j} G_{k}=-G_{k} G_{j}(j \neq k) .
$$

We can write our Dirac operator as

$$
\mathcal{D}=G_{0}\left(\partial_{u}+B\right)
$$

where

$$
B=A_{1} v_{1} D_{v_{1}}+\cdots+A_{\ell} v_{\ell} D_{v_{\ell}} \in \operatorname{Diff}_{b}^{1}\left(\overline{\mathbb{R}}_{+}^{\ell}, E\right)
$$

with $A_{j}=i G_{j} G_{0}$ and $D_{v_{j}}=i^{-1} \partial_{v_{j}}$. The properties (3.1) of the $G_{j}$ 's imply that

$$
A_{j}^{2}=\mathrm{Id}, A_{j}^{*}=A_{j}, A_{j} A_{k}=-A_{k} A_{j} \quad, \quad \text { where } A_{j}=i G_{j} G_{0} .
$$

These properties imply, in particular, that $B$ is formally self-adjoint.

To find the orthogonalized Calderón projector we first determine the Cauchy data space of $\mathcal{D}$. To this end, let $\phi(u, v) \in H_{b}^{\infty}\left(X_{R}, E\right)$. We first write $\phi$ in terms of the Mellin transform

$$
\phi(u, v)=\int_{\mathbb{R}^{\ell}} v^{i \tau} \hat{\phi}(u, \tau) d \tau,
$$

where the " ' ' means Mellin transform with respect to $v$ :

$$
\hat{\phi}(u, \tau)=\int_{\overline{\mathbb{R}}_{+}^{e}} v^{-i \tau} \phi(u, v) \frac{d v}{v} .
$$

Second, we note that

$$
\mathcal{D} \phi=G_{0}\left(\partial_{u}+B\right) \int v^{i \tau} \hat{\phi}(u, \tau) d \tau=G_{0} \int v^{i \tau}\left(\partial_{u}+A(\tau)\right) \hat{\phi}(u, \tau) d \tau,
$$

where recalling that $B=A_{1} v_{1} D_{v_{1}}+\cdots+A_{\ell} v_{\ell} D_{v_{\ell}}$, we have

$$
A(\tau):={ }^{b} \sigma_{1}(B)(\tau)=A_{1} \tau_{1}+\cdots+A_{\ell} \tau_{\ell} .
$$

Thus, $\mathcal{D} \phi=0$ if and only if $\left(\partial_{u}+A(\tau)\right) \hat{\phi}(u, \tau)=0$, if and only if

$$
\hat{\phi}(u, \tau)=e^{-u A(\tau)} \hat{\phi}(0, \tau) .
$$

With this formula in mind, for $\varphi \in H_{b}^{\infty}\left(\overline{\mathbb{R}}_{+}^{\ell}, E\right)$, we define the operator $e^{-u B} \varphi$ via the Mellin transform:

$$
e^{-u B} \varphi:=\int v^{i \tau} e^{-u A(\tau)} \hat{\varphi}(\tau) d \tau,
$$

provided that the right-hand side exists. Then $\mathcal{D} \phi=0$ if and only if

$$
\phi(u, v)=e^{-u B} \varphi \quad, \quad \varphi(v)=\phi(0, v) .
$$


We can now find the Cauchy data space. Recall that $X_{R}=[0, R] \times \overline{\mathbb{R}}_{+}^{\ell}$ and $Y_{R}=\partial X_{R}=\overline{\mathbb{R}}_{+}^{\ell} \sqcup \overline{\mathbb{R}}_{+}^{\ell}$. Then $H_{b}^{\infty}\left(Y_{R}, E\right) \equiv H_{b}^{\infty}\left(\overline{\mathbb{R}}_{+}^{\ell}, E\right) \oplus H_{b}^{\infty}\left(\overline{\mathbb{R}}_{+}^{\ell}, E\right)$ and with respect to this decomposition, we have

$$
\left.\phi\right|_{Y_{R}}=\left(\begin{array}{c}
\left.\phi\right|_{u=0} \\
\left.\phi\right|_{u=R}
\end{array}\right)=\left(\begin{array}{c}
\varphi \\
e^{-R B} \varphi
\end{array}\right)
$$

In conclusion, we have proved that if $\phi \in H_{b}^{\infty}\left(X_{R}, E\right)$ and $\mathcal{D} \phi=0$, then $\left.\phi\right|_{Y_{R}}$ is a column vector $\left(\varphi, e^{-R B} \varphi\right)$ with $\varphi, e^{-R B} \varphi \in H_{b}^{\infty}\left(\overline{\mathbb{R}}_{+}^{\ell}, E\right)$. The converse is straightforward (see the structure of $A(\tau)$ in (3.3) below.) Thus, we have proved

Theorem 3.1. The Cauchy data space

$$
\mathcal{H}(\mathcal{D}):=\left\{\left.\phi\right|_{Y_{R}} \mid \phi \in H_{b}^{\infty}\left(X_{R}, E\right), \mathcal{D} \phi=0\right\} \subset H_{b}^{\infty}\left(\overline{\mathbb{R}}_{+}^{\ell}, E\right) \oplus H_{b}^{\infty}\left(\overline{\mathbb{R}}_{+}^{\ell}, E\right)
$$

is given explicitly by

$$
\mathcal{H}(\mathcal{D})=\left\{\left(\begin{array}{c}
\mathrm{Id} \\
e^{-R B}
\end{array}\right) \varphi \mid \varphi \in H_{b}^{\infty}\left(\overline{\mathbb{R}}_{+}^{\ell}, E\right), e^{-R B} \varphi \in H_{b}^{\infty}\left(\overline{\mathbb{R}}_{+}^{\ell}, E\right)\right\} .
$$

In order to determine the properties of the orthogonalized Calderón projector, we first note that

$$
A(\tau)^{*}=A(\tau) \quad, \quad A(\tau)^{2}=|\tau|^{2} \quad, \quad A(\tau) G_{0}=-G_{0} A(\tau) .
$$

These properties follow directly from (3.2) or from the fact that $A(\tau)={ }^{b} \sigma_{1}(B)(\tau)$. It follows that $A(\tau)$ has eigenvalues $\pm|\tau|$ with the corresponding eigenspaces of the same dimension which $G_{0}$ intertwines. In particular, for any $u \in \mathbb{R}$,

$$
e^{-u A(\tau)} \text { acts as } e^{\mp u|\tau|} \text { where } A(\tau)= \pm|\tau| \text {. }
$$

From this fact, it is easy to show that for $\varphi \in H_{b}^{\infty}\left(\overline{\mathbb{R}}_{+}^{\ell}, E\right)$, we have $e^{-R B} \varphi \in$ $H_{b}^{\infty}\left(\overline{\mathbb{R}}_{+}^{\ell}, E\right)$ if and only if for any $k$,

$$
\int e^{2 R|\tau|}|\tau|^{k}|\hat{\varphi}(\tau)|^{2} d \tau<\infty \quad \text { for all } k .
$$

Now let us compute the orthogonalized Calderón projector:

Theorem 3.2. The orthogonal projector onto the Cauchy data space $\mathcal{H}(\mathcal{D})$ is the pseudodifferential operator $\mathcal{C} \in \widetilde{\Psi}_{b}^{0}(Y, E)$ given by (cf. (2.6))

$$
\mathcal{C}=\frac{1}{\operatorname{Id}+e^{-2 R B}}\left(\begin{array}{cc}
\operatorname{Id} & e^{-R B} \\
e^{-R B} & e^{-2 R B}
\end{array}\right):=\int_{\mathbb{R}^{\ell}}\left(\frac{v}{v^{\prime}}\right)^{i \tau} c(\tau) d \tau \cdot \frac{d v^{\prime}}{v^{\prime}},
$$

where

$$
c(\tau)=\frac{1}{\operatorname{Id}+e^{-2 R A(\tau)}}\left(\begin{array}{cc}
\operatorname{Id} & e^{-R A(\tau)} \\
e^{-R A(\tau)} & e^{-2 R A(\tau)}
\end{array}\right) .
$$

Moreover, the b-principal symbol of $\mathcal{C}$ is given by

$$
{ }^{b} \sigma_{0}(\mathcal{C})(\tau)=\left(\begin{array}{cc}
\Pi_{+}(A(\tau)) & 0 \\
0 & \Pi_{-}(A(\tau))
\end{array}\right)
$$

where $\Pi_{ \pm}(A(\tau))$ are the orthogonal projectors onto the $\pm|\tau|$ eigenspaces of the symbol $A(\tau)={ }^{b} \sigma_{1}(B)(\tau)$ of the tangential operator. 
Proof. We first show that $\mathcal{C}$ is a $b$-pseudodifferential operator and then we prove that $\mathcal{C}$ does indeed project onto $\mathcal{H}(\mathcal{D})$. First, we note that $c(\tau)$ is smooth in all variables and we can write

$$
c(\tau)=\left(\begin{array}{cc}
\frac{1}{\mathrm{Id}+e^{-2 R A(\tau)}} & \frac{1}{e^{R A(\tau)}+e^{-R A(\tau)}} \\
\frac{1}{e^{R A(\tau)}+e^{-R A(\tau)}} & \frac{1}{e^{2 R A(\tau)}+\mathrm{Id}}
\end{array}\right) .
$$

From this expression, a straightforward verification from (3.4) shows that $c(\tau)$ is a classical symbol of order 0 . Another straightforward verification shows that $c(\tau)$ satisfies the lacunary condition on a strip $|\operatorname{Im} \tau| \leq \delta$ for some $\delta>0$ (cf. the discussion after the proof). Second, we see that over the space where $A(\tau)= \pm|\tau|$, we have

$$
\begin{aligned}
c(\tau) & =\left(\begin{array}{cc}
\frac{1}{\mathrm{Id}+e^{\mp 2 R|\tau|}} & \frac{1}{e^{ \pm R|\tau|}+e^{\mp R|\tau|}} \\
\frac{1}{e^{ \pm R|\tau|}+e^{\mp R|\tau|}} & \frac{1}{e^{ \pm 2 R|\tau|}+\mathrm{Id}}
\end{array}\right) \\
& =\left(\begin{array}{cc}
\Pi_{+}(A(\tau)) & 0 \\
0 & \Pi_{-}(A(\tau))
\end{array}\right)+\text { exponentially decreasing as }|\tau| \rightarrow \infty .
\end{aligned}
$$

This shows that

$$
{ }^{b} \sigma_{0}(\mathcal{C})(\tau)=\left(\begin{array}{cc}
\Pi_{+}(A(\tau)) & 0 \\
0 & \Pi_{-}(A(\tau))
\end{array}\right) .
$$

Third, by direct computation, we see that

$$
\begin{aligned}
& c(\tau)^{2}=\frac{1}{\left(\operatorname{Id}+e^{-2 R A(\tau)}\right)^{2}}\left(\begin{array}{cc}
\operatorname{Id} & e^{-R A(\tau)} \\
e^{-R A(\tau)} & e^{-2 R A(\tau)}
\end{array}\right)\left(\begin{array}{cc}
\operatorname{Id} & e^{-R A(\tau)} \\
e^{-R A(\tau)} & e^{-2 R A(\tau)}
\end{array}\right) \\
& =\frac{1}{\operatorname{Id}+e^{-2 R A(\tau)}}\left(\begin{array}{cc}
\operatorname{Id} & e^{-R A(\tau)} \\
e^{-R A(\tau)} & e^{-2 R A(\tau)}
\end{array}\right)=c(\tau) .
\end{aligned}
$$

Since $c(\tau)$ is certainly self-adjoint, we conclude that

$$
\mathcal{C}^{2}=\mathcal{C} \text { and } \mathcal{C}^{*}=\mathcal{C} .
$$

Thus, we have proven that $\mathcal{C} \in \widetilde{\Psi}_{b}^{0}(Y, E)$ and is an orthogonal projection. It remains to show that $\mathcal{C}$ defines the projection onto $\mathcal{H}(\mathcal{D})$. To this end, let $(\varphi, \psi) \in$ $H_{b}^{\infty}(Y, E)$. Then by definition,

$$
\begin{aligned}
\mathcal{C}(\varphi, \psi) & =\int v^{i \tau} \frac{1}{\operatorname{Id}+e^{-2 R A(\tau)}}\left(\begin{array}{cc}
\operatorname{Id} & e^{-R A(\tau)} \\
e^{-R A(\tau)} & e^{-2 R A(\tau)}
\end{array}\right)\left(\begin{array}{c}
\hat{\varphi}(\tau) \\
\hat{\psi}(\tau)
\end{array}\right) d \tau \\
& =\int e^{i v \tau}\left(\begin{array}{c}
\operatorname{Id} \\
e^{-R A(\tau)}
\end{array}\right) \hat{\omega}(\tau) d \tau
\end{aligned}
$$

where

$$
\omega(v)=\int v^{i \tau} \frac{\hat{\varphi}(\tau)+e^{-R A(\tau)} \hat{\psi}(\tau)}{\operatorname{Id}+e^{-2 R A(\tau)}} d \tau .
$$

Using (3.4), one sees that (3.5) is satisfied, so by Theorem 3.1, $\mathcal{C}$ has image in the Cauchy data space $\mathcal{H}(\mathcal{D})$. Also, this computation shows that $\mathcal{C}=\operatorname{Id}$ on $\mathcal{H}(\mathcal{D})$, so the image of $\mathcal{C}$ is exactly $\mathcal{H}(\mathcal{D})$ and our proof is complete.

We remark that in general, $\mathcal{C}$ is not in the small $b$-calculus. This is because the operator $\left(\operatorname{Id}+e^{-2 R A(\tau)}\right)^{-1}$ will always have poles so the symbol $c(\tau)$ of $\mathcal{C}$ is not entire. For example, let us consider the case $\ell=1$ when $X_{R}=[0, R] \times \overline{\mathbb{R}}_{+}$. In 
this case, we have $A(\tau)=\tau A_{1}$ where $A_{1}=i G_{1} G_{0}$ satisfies $A_{1}^{*}=A_{1}$ and $A_{1}^{2}=\mathrm{Id}$. Thus, with respect to the decomposition into the \pm 1 eigenspaces of $A_{1}$, we have

$$
\frac{1}{\mathrm{Id}+e^{-2 R A(\tau)}}=\frac{1}{\mathrm{Id}+e^{\mp 2 R \tau}} \quad \text { on the } \pm 1 \text { eigenspaces of } A_{1} .
$$

In particular, the function $\mathrm{Id}+e^{\mp 2 R \tau}$ vanishes when $\tau= \pm i \pi /(2 R)$. Further, this equation shows that $c(\tau)$ satisfies the lacunary condition only on the strip $|\operatorname{Im} \tau|<\pi /(2 R)$. Thus, when $\ell=1$, we can say precisely that $\mathcal{C} \in \Psi_{b}^{0, \theta}(Y, E)$ for any fixed $0<\theta<\pi /(2 R)$.

We end this section with the $A P S$ spectral projector. Back to the general case $X_{R}=[0, R] \times \overline{\mathbb{R}}_{+}^{\ell}$. Since $\mathcal{D}=G_{0}\left(\partial_{u}+B\right)$, the induced Dirac operator on $Y=$ $\partial X_{R}=\overline{\mathbb{R}}_{+}^{\ell} \sqcup \overline{\mathbb{R}}_{+}^{\ell}$ is

$$
D_{0}=\left(\begin{array}{cc}
-B & 0 \\
0 & B
\end{array}\right)
$$

Recalling that $B=A_{1} v_{1} D_{v_{1}}+\cdots+A_{\ell} v_{\ell} D_{v_{\ell}}$ we see that the nonpositive spectral projector of $D_{0}$ is the operator

$$
\Pi_{A P S}:=\int_{\mathbb{R}^{\ell}}\left(\frac{v}{v^{\prime}}\right)^{i \tau}\left(\begin{array}{cc}
\Pi_{+}(A(\tau)) & 0 \\
0 & \Pi_{-}(A(\tau))
\end{array}\right) d \tau \frac{d v^{\prime}}{v^{\prime}} .
$$

Note that the complete symbol of $\Pi_{A P S}$, which is just the $b$-principal symbol ${ }^{b} \sigma_{0}(\mathcal{C})$ of the orthogonalized Calderón projector $\mathcal{C}$, is not smooth at $\tau=0$, so $\Pi_{A P S}$ is not a pseudodifferential operator.

\section{The invertible EXtension}

In this section, we construct an extension of $\mathcal{D}$, which is an invertible Fredholm Dirac operator. This extension will be used to construct the Calderón projector and derive its properties.

Theorem 4.1. There exists a smooth manifold $\widetilde{X}$ with corners that contains $X$ as a submanifold and an invertible Fredholm Dirac type operator $\widetilde{\mathcal{D}} \in \operatorname{Diff}_{b}^{1}(\widetilde{X}, \widetilde{E}, \widetilde{F})$ such that $\left.\widetilde{\mathcal{D}}\right|_{X}=\mathcal{D}$.

This theorem generalizes Wojciechowski's [32] (cf. Chapter 9 of [5]) result for smooth manifolds with boundary. Note that this theorem holds regardless of the dimension of $X$ and with no invertibility assumptions on $\mathcal{D}$. To prove this theorem, we first reduce our theorem to the formally self-adjoint case. To do so, consider the vector bundle $E^{\prime}:=E \oplus F$ over $X$ and the Dirac type operator

$$
\mathcal{D}^{\prime}:=\left(\begin{array}{cc}
0 & \mathcal{D}^{*} \\
\mathcal{D} & 0
\end{array}\right): C^{\infty}\left(X, E^{\prime}\right) \rightarrow C^{\infty}\left(X, E^{\prime}\right) .
$$

This operator is by construction formally self-adjoint and it still satisfies the collar decomposition property (1.6). If we prove our theorem for this operator, then we automatically get an invertible extension for $\mathcal{D}$. Thus, for the rest of this section, we assume that $\mathcal{D}: C^{\infty}(X, E) \rightarrow C^{\infty}(X, E)$ is formally self-adjoint.

Next, following Booß-Bavnbek and Wojciechowski, Chapter 9 of [5], we reduce our problem to the product case near $H_{0}$. To this end, by (1.6) we know that over the collar neighborhood $[-1,0] \times H_{0}$ of $H_{0}$, we can write

$$
\mathcal{D}=G_{u}\left(\partial_{u}+D_{u}\right)
$$




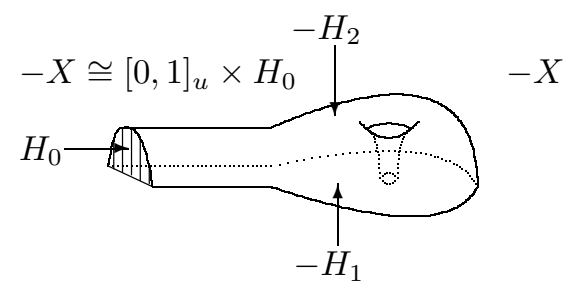

Figure 2. $-X$ is the 'mirror image' of the original manifold $X$.

where $G_{u}$ is a family of unitary maps on $E_{0}:=\left.E\right|_{H_{0}}$, and $D_{u}$ is a family of operators in $\operatorname{Diff}_{b}^{1}\left(H_{0}, E_{0}\right)$ depending smoothly on $u$. Attaching the collar $[0,2]_{u} \times H_{0}$ to $H_{0}$ we can form the manifold

$$
X \sqcup\left([0,2]_{u} \times H_{0}\right),
$$

then it is straightforward to extend $\mathcal{D}$ to this manifold in such a way that $\mathcal{D}$ is independent of $u$ on $[1,2]_{u} \times H_{0}$ :

$$
\mathcal{D}=G_{0}\left(\partial_{u}+D_{0}\right)
$$

where $G_{0}$ is a unitary map on $E_{0}$ and $D_{0} \in \operatorname{Diff}_{b}^{1}\left(H_{0}, E_{0}\right)$ is formally self-adjoint. By proving Theorem 4.1 for this extended operator, we may assume that $\mathcal{D}$ takes the product form (4.1) on the original neighborhood $N=[-1,0] \times H_{0}$, which we now assume. Then using the fact that $\mathcal{D}$ is of Dirac type and is formally self-adjoint, we observe that

$$
G_{0}^{2}=-\mathrm{Id} \quad \text { and } \quad D_{0} G_{0}=-G_{0} D_{0} .
$$

With this product structure fixed, we proceed to construct the invertible double of the Dirac type operator $\mathcal{D}$.

4.1. The doubled manifold. We begin by defining the manifold $-X$ to be the same manifold $X$ but on the collar $N=[-1,0]_{u} \times Y$, we simply make the change of variables $u \mapsto-u$ so that near $H_{0}$ (see Figure 2)

$$
-X \cong-N:=[0,1]_{u} \times H_{0} .
$$

The minus sign just indicates that we always use the collar $-N$ near $H_{0}$ on $-X$ instead of $N$, which in the literature is sometimes stated as ' $-X$ is $X$ with the reversed orientation'. Since $H_{0}$ is admissible, all the hypersurfaces intersect $H_{0}$, therefore each of the hypersurfaces $H_{j}$ with $j>0$ are also reversed as seen in Figure 2. Of course, since $X \equiv-X$, the vector bundle $E$ is still a vector bundle over $-X$ and $\mathcal{D}$ defines an operator on $C^{\infty}(-X, E)$. The only difference is that on $-X$ we use the collar $-N$, and since $\mathcal{D}$ is of the form (4.1) over $N$, under the change of variables $u \mapsto-u$, we have

$$
\mathcal{D}=G_{0}\left(-\partial_{u}+D_{0}\right) \quad \text { over } \quad-N=[0,1]_{u} \times H_{0}
$$

We now glue $X$ to $-X$ via (see Figure 3 )

$$
\widetilde{X}:=X \sqcup_{H_{0}}(-X) \quad \text { and } \quad \widetilde{E}:=E \sqcup_{G_{0}} E,
$$

which means that $X$ and $-X$ are attached along $H_{0}$ and $\widetilde{E}$ is obtained by gluing $E$ on $X$ to $E$ on $-X$ via the relation $e \sim G_{0} e$ over $H_{0}$. The $C^{\infty}$ structure of $\widetilde{E}$ is 


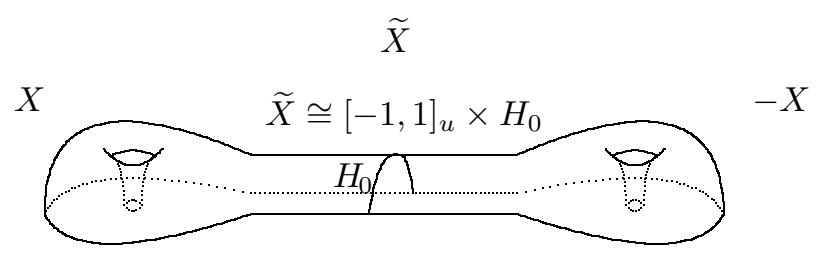

Figure 3 . In this example, $\widetilde{X}$ is the double of the manifold on the left side in Figure 1. The hypersurfaces $H_{1}$ and $H_{2}$ automatically get doubled in the process.

defined as follows. Since $\widetilde{E} \equiv E$ over $X$ and $-X$ away from $H_{0}$, we can focus near the gluing hypersurface $H_{0}$. Since over $N$,

$$
\left.E\right|_{N} \cong\left([-1,0] \times H_{0}, E_{0}\right),
$$

over $-N=[0,1] \times H_{0}$, we have

$$
\left.E\right|_{-N} \cong\left([0,1] \times H_{0}, E_{0}\right) \stackrel{G_{0}}{\longrightarrow}\left([0,1] \times H_{0}, E_{0}\right),
$$

where $G_{0}$ is an isomorphism on $E_{0}$. Let $\widetilde{N}:=N \sqcup_{H_{0}}(-N)$. Then by definition of $\widetilde{E}$, using (4.5) and (4.6) we get a bijection

$$
\left.\widetilde{E}\right|_{\widetilde{N}}:=\left(\left.E\right|_{N}\right) \sqcup_{G_{0}}\left(\left.E\right|_{-N}\right) \longleftrightarrow\left([-1,1] \times H_{0}, E_{0}\right) .
$$

We define the $C^{\infty}$ structure of $\widetilde{E}$ near $H_{0}$ by simply declaring this bijection to be a diffeomorphism. Now working out the definition of the $C^{\infty}$ structure of $\widetilde{E}$, we see that an element of $C^{\infty}(\widetilde{X}, \widetilde{E})$ can be identified as a pair

$$
\phi=\left(\phi_{1}, \phi_{2}\right) \quad, \quad \text { where } \phi_{1} \in C^{\infty}(X, E), \phi_{2} \in C^{\infty}(-X, E),
$$

such that in the trivializations $\left.E\right|_{N} \cong\left([-1,0]_{u} \times H_{0}, E_{0}\right)$ and $\left.E\right|_{-N} \cong\left([0,1]_{u} \times\right.$ $\left.H_{0}, E_{0}\right)$, the following section

$$
\phi(u, y):= \begin{cases}\phi_{1}(u, y) & u<0 \\ G_{0} \phi_{2}(u, y) & u>0\end{cases}
$$

extends to $u=0$ to define a smooth section $\phi(u, y) \in C^{\infty}\left([-1,1]_{u} \times H_{0}, E_{0}\right)$.

It is important to note that, by the admissibility of $H_{0}$, if $H_{j}$ with $j>0$ is any boundary hypersurface, then the hypersurfaces $H_{j}$ and $-H_{j}$ glue via (4.4) to form a hypersurface $\widetilde{H}_{j}$ in $\widetilde{X}$ such that (see Figure 3 )

$$
\widetilde{H}_{j}=H_{j} \sqcup_{H_{0} \cap H_{j}}\left(-H_{j}\right) \quad \text { and }\left.\quad \widetilde{E}\right|_{\widetilde{H}_{j}}:=\left.\left.E\right|_{H_{j}} \sqcup_{G_{0}} E\right|_{H_{j}} .
$$

Moreover, $\left\{\widetilde{H}_{j}\right\}(j>0)$ are all the boundary hypersurfaces of $\widetilde{X}$ and the boundary defining functions $\left\{x_{j}\right\}$ for $\left\{H_{j}\right\}$ induce corresponding boundary defining functions $\left\{\tilde{x}_{j}\right\}$ for the $\left\{\widetilde{H}_{j}\right\}$, and the decomposition (1.1) continues to hold near $\widetilde{H}_{i}$ :

$$
\widetilde{X} \cong[0,1]_{\tilde{x}_{i}} \times \widetilde{H}_{i}, \quad i=1,2, \ldots, r \quad, \quad \widetilde{H}_{i}=\left\{\tilde{x}_{i}=0\right\} .
$$

There are also decompositions of the sort (2.10): If $\widetilde{Y}$ is a component of $\widetilde{H}_{i_{1}} \cap \cdots \cap$ $\widetilde{H}_{i_{\ell}}$, where $1 \leq i_{1}<\cdots<i_{\ell}$, then near $\widetilde{Y}$,

$$
\tilde{X} \cong[0,1]_{\tilde{v}}^{\ell} \times \tilde{Y} \quad, \quad \tilde{v}=\left(\tilde{x}_{i_{1}}, \ldots, \tilde{x}_{i_{\ell}}\right) .
$$


Note that $\tilde{Y}$ is just the double of a component in $H_{i_{1}} \cap \cdots \cap H_{i_{\ell}}$. Finally, the metric $g$ in (1.3) becomes an exact $b$-metric $\tilde{g}$ on $\widetilde{X}$ that pushes all its faces $\left\{\widetilde{H}_{j}\right\}$ to $\infty$ :

$$
\tilde{g}=\sum_{i=1}^{r}\left(\frac{d \tilde{x}_{i}}{\tilde{x}_{i}}\right)^{2}+\tilde{h},
$$

where $\tilde{h}$ is a smooth symmetric two-form on $\tilde{X}$.

4.2. The invertible double. We now define the doubled Dirac operator $\widetilde{\mathcal{D}}$ acting on $C^{\infty}(\widetilde{X}, \widetilde{E})$ of the Dirac type operator $\mathcal{D}$. Given a section $\phi=\left(\phi_{1}, \phi_{2}\right) \in$ $C^{\infty}(\widetilde{X}, \widetilde{E})$, we define

$$
\widetilde{\mathcal{D}} \phi:=\left(\mathcal{D} \phi_{1},-\mathcal{D} \phi_{2}\right)
$$

We need to verify that $\left(\mathcal{D} \phi_{1},-\mathcal{D} \phi_{2}\right) \in C^{\infty}(\tilde{X}, \widetilde{E})$. To prove this, in view of $(4.7)$, we need to show that $\mathcal{D} \phi_{1}$ and $-G_{0} \mathcal{D} \phi_{2}$ patch near $H_{0}$ to be smooth. To this end, we note that since $\mathcal{D}=G_{0}\left(\partial_{u}+D_{0}\right)$ over $N$, we have

$$
\mathcal{D} \phi_{1}(u, y)=G_{0}\left(\partial_{u}+D_{0}\right) \phi(u, y) \text {. }
$$

From (4.2) we know that $G_{0}^{2}=-\mathrm{Id}$ and $D_{0} G_{0}=-G_{0} D_{0}$. Thus, since $\mathcal{D}=$ $G_{0}\left(-\partial_{u}+D_{0}\right)$ over $-N$, again recalling (4.7), we have

$$
\begin{aligned}
-G_{0} \mathcal{D}\left(\phi_{2}\right)=-G_{0} \cdot\left(G_{0}\left(-\partial_{u}+D_{0}\right)\right)\left(-G_{0} \phi\right) & =-\left(-\partial_{u}+D_{0}\right) G_{0} \phi \\
& =G_{0}\left(\partial_{u}+D_{0}\right) \phi .
\end{aligned}
$$

Comparing the formulas for $\mathcal{D} \phi_{1}(u, y)$ and $-G_{0} \mathcal{D} \phi_{2}(u, y)$, we see that

$$
\widetilde{\mathcal{D}} \phi=G_{0}\left(\partial_{u}+D_{0}\right) \phi(u, y) \quad \text { over } \quad \tilde{N}=[-1,1]_{u} \times H_{0} .
$$

Thus, $\widetilde{\mathcal{D}}: C^{\infty}(\widetilde{X}, \widetilde{E}) \rightarrow C^{\infty}(\widetilde{X}, \widetilde{E})$ takes the product form (4.10) near $H_{0}$. Since $\mathcal{D}$ is a $b$-operator, the definition (4.9) shows that $\widetilde{\mathcal{D}}$ is a $b$-differential operator away from $H_{0}$ and then the form (4.10) shows that $\widetilde{\mathcal{D}}$ is a $b$-operator near $H_{0}$. Hence, $\widetilde{\mathcal{D}} \in \operatorname{Diff}_{b}^{1}(\widetilde{X}, \widetilde{E})$. Note that $\widetilde{E}$ inherits an inner product from $E$, therefore $L_{b}^{2}(\widetilde{X}, \widetilde{E})$ has a naturally induced inner product. Since $\mathcal{D}$ is by assumption formally self-adjoint, the definition (4.9) and the product form (4.10) shows that $\widetilde{\mathcal{D}}$ is also formally self-adjoint.

4.3. Fredholmness of the double. We now show that $\widetilde{\mathcal{D}}$ is of Dirac type and Fredholm. The definition (4.9) and the decomposition (4.10) show that ${ }^{b} \sigma_{1}(\widetilde{\mathcal{D}})^{2}=$ $\tilde{g}$, so it remains to verify the structure of $\widetilde{\mathcal{D}}$ near the faces of $\widetilde{X}$. Consider the decomposition of $\widetilde{X}$ near $\widetilde{Y}$ as in (4.8), and let $X \cong[0,1]_{v}^{\ell} \times Y$ be the corresponding decomposition of $X$ near $Y$. Then by (2.14), near $Y$ we can write

$$
\mathcal{D}=G_{1} v_{1} \partial_{v_{1}}+\cdots+G_{\ell} v_{\ell} \partial_{v_{\ell}}+B_{Y}+\mathcal{O}(v)
$$

where $B_{Y} \in \operatorname{Diff}_{b}^{1}\left(Y, E_{Y}\right)$ with $E_{Y}:=\left.E\right|_{Y}, \mathcal{O}(v)$ is a first order b-differential operator that vanishes at $Y$, and where the $G_{j}$ 's are unitary maps on $E_{Y}$ satisfying the relations

$$
G_{j}^{2}=-\mathrm{Id} \quad, \quad G_{j} G_{k}=-G_{k} G_{j}(j \neq k) \quad, \quad G_{j} B_{Y}=-B_{Y} G_{j}
$$


Note that these relations are slightly different from $(2.15)$ because now $\mathcal{D}$ is formally self-adjoint so we have more structure forced upon us. It follows that on the decomposition (4.8) near $\widetilde{Y}, \widetilde{X} \cong[0,1]_{\tilde{v}}^{\ell} \times \tilde{Y}$, we have

$$
\widetilde{\mathcal{D}}=\widetilde{G}_{1} \tilde{v}_{1} \partial_{\tilde{v}_{1}}+\cdots+\widetilde{G}_{\ell} \tilde{v}_{\ell} \partial_{\tilde{v}_{\ell}}+\widetilde{B}_{\widetilde{Y}}+\mathcal{O}(\tilde{v})
$$

where the operators $\widetilde{G}_{j}$ and $\widetilde{B}_{\widetilde{Y}}$ are defined on sections on $\widetilde{E}_{\widetilde{Y}}:=\left.\widetilde{E}\right|_{\widetilde{Y}}$ over the double of $Y$ by corresponding formulas to (4.9):

$$
\widetilde{G}_{j} \phi:=\left(G_{j} \phi_{1},-G_{j} \phi_{2}\right) \quad, \quad \widetilde{B}_{\widetilde{Y}} \phi:=\left(B_{Y} \phi_{1},-B_{Y} \phi_{2}\right) .
$$

We know that $\widetilde{\mathcal{D}}$ maps smooth sections of $\widetilde{E}$ to itself, but it is not a priori automatic that $\widetilde{G}_{j}$ and $\widetilde{B}_{\widetilde{Y}}$ individually map smooth sections of $\widetilde{E}_{\widetilde{Y}}$ to itself. We prove this is so in the following lemma.

Lemma 4.2. The operators $\widetilde{G}_{j}$ and $\widetilde{B}_{\widetilde{Y}}$ define operators on $C^{\infty}\left(\widetilde{Y}, \widetilde{E}_{\widetilde{Y}}\right)$ and they satisfy the properties (4.11) with 'tildes'. In particular, $\widetilde{\mathcal{D}}$ is a Dirac type operator over $\widetilde{X}$.

Proof. Taking the normal operator of $\widetilde{\mathcal{D}}$, we see that

$$
N_{\widetilde{Y}}(\widetilde{\mathcal{D}})(\tau):=\left.\left(\tilde{v}^{-i \tau} \widetilde{\mathcal{D}} \tilde{v}^{i \tau}\right)\right|_{\widetilde{Y}}=\widetilde{G}_{1} i \tau_{1}+\cdots+\widetilde{G}_{\ell} i \tau_{\ell}+\widetilde{B}_{\widetilde{Y}}
$$

which by definition is a smooth family of $b$-operators on $\tilde{Y}$. In particular, setting $\tau=0$ shows that $\widetilde{B}_{\widetilde{Y}}$ maps $C^{\infty}\left(\widetilde{Y}, \widetilde{E}_{\widetilde{Y}}\right)$ to itself. Then fixing $j$ and setting $\tau_{k}=0$ for $k \neq j$ implies that $\widetilde{G}_{j}$ maps $C^{\infty}\left(\widetilde{Y}, \widetilde{E}_{\widetilde{Y}}\right)$ to itself. The properties (4.11) are straightforward to verify; for instance,

$$
\begin{aligned}
\widetilde{G}_{k} \widetilde{B}_{\widetilde{Y}} \phi=\widetilde{G}_{k}\left(B_{Y} \phi_{1},-B_{Y} \phi_{2}\right) & =\left(G_{k} B_{Y} \phi_{1}, G_{k} B_{Y} \phi_{2}\right) \\
& =-\left(B_{Y} G_{k} \phi_{1}, B_{Y} G_{k} \phi_{2}\right) \\
& =-\widetilde{B}_{\widetilde{Y}}\left(G_{k} \phi_{1},-G_{k} \phi_{2}\right)=-\widetilde{B}_{\widetilde{Y}} \widetilde{G}_{k} \phi
\end{aligned}
$$

We now prove that $\widetilde{\mathcal{D}}$ is Fredholm. By Theorem 2.5 we need to show that $\widetilde{B}_{\widetilde{Y}}$ has $L_{b}^{2}$ kernel 0 . So assume that $\widetilde{B}_{\widetilde{Y}}\left(\phi_{1}, \phi_{2}\right)=\left(B_{Y} \phi_{1},-B_{Y} \phi_{2}\right)=0$. This implies that $B_{Y} \phi_{1}=0=B_{Y} \phi_{2}$. Since $\mathcal{D}=G_{0}\left(\partial_{u}+D_{0}\right)$ near $H_{0}$, we see that

$$
B_{Y}=\left.\mathcal{D}\right|_{Y}=G_{Y}\left(\partial_{u}+D_{Y}\right) \quad \text { over } \quad N \cap Y=[-1,0]_{u} \times H_{0} \cap Y,
$$

where $G_{Y}=\left.G_{0}\right|_{Y}$ and $D_{Y}=\left.D_{0}\right|_{Y}$. Therefore we can apply Green's formula (2.18) on $Y$ to get

$$
\begin{aligned}
0=0-0=\left\langle B_{Y} \phi_{1}, \phi_{2}\right\rangle-\left\langle\phi_{1}, B_{Y} \phi_{2}\right\rangle & =\left\langle\left. G_{Y} \phi_{1}\right|_{Y},\left.\phi_{2}\right|_{Y}\right\rangle_{Y} \\
& =-\left\langle\left.\phi_{2}\right|_{Y},\left.\phi_{2}\right|_{Y}\right\rangle_{Y}=-\left\|\left.\phi_{2}\right|_{Y}\right\|_{Y}^{2},
\end{aligned}
$$

where we used that $\left.\phi_{1}\right|_{Y}=G_{Y} \phi_{2}$. This implies that $\left.\phi_{2}\right|_{Y}=0$ and thus $\left.\phi_{1}\right|_{Y}=$ $\left.G_{Y} \phi_{2}\right|_{Y}=0$. Thus, with $B_{Y} \phi_{1}=0=B_{Y} \phi_{2}$ and the unique continuation theorem, which also holds for our manifold [1, Cor. 1], we get $\phi_{1}=0=\phi_{2}$. Thus, $B_{Y}$ has $L_{b}^{2}$ kernel 0 , so by Theorem 2.5, $\widetilde{\mathcal{D}}$ is Fredholm. The same argument that we just did also shows that $\widetilde{\mathcal{D}}$ has $L_{b}^{2}$ kernel 0 . Thus, $\widetilde{\mathcal{D}}$ is invertible and our proof of Theorem 4.1 is complete. 


\section{The Calderón Projector}

In this section we prove Theorems 1.1, 1.2 and 1.3.

5.1. Proof of Theorem 1.1. First let us show that

$$
\mathcal{D}: H_{b}^{\infty}(X, E) \rightarrow H_{b}^{\infty}(X, F)
$$

is surjective. Let $\psi \in H_{b}^{\infty}(X, F)$ and choose any element $\tilde{\psi} \in H_{b}^{\infty}(\widetilde{X}, \widetilde{F})$ with $\left.\tilde{\psi}\right|_{X}=\psi$ where $\widetilde{X}$ is the manifold defined in (4.4). Then set

$$
\phi:=\left.\left(\widetilde{\mathcal{D}}^{-1} \widetilde{\psi}\right)\right|_{X},
$$

where $\widetilde{\mathcal{D}}$ is the invertible extension constructed in subsection 4.2. By definition, it is obvious that $\mathcal{D} \phi=\psi$. By the unique continuation principle [1, Cor. 1], the restriction of $\operatorname{ker} \mathcal{D}$ to the boundary gives a canonical isomorphism between ker $\mathcal{D}$ and $\mathcal{H}(\mathcal{D})$. Now using the invertible extension $\widetilde{\mathcal{D}}$, let us define

$$
P:=\lim _{\varepsilon \rightarrow 0^{-}}-\gamma_{\varepsilon} \widetilde{\mathcal{D}}^{-1} \gamma_{0}^{*} G_{0}: H_{b}^{\infty}\left(H_{0}, E_{0}\right) \rightarrow H_{b}^{\infty}\left(H_{0}, E_{0}\right),
$$

where $\gamma_{\varepsilon}$ is restriction to $\{\varepsilon\} \times H_{0}$ and $\gamma_{0}^{*}$ is the adjoint map of $\gamma_{0}$ at $\{0\} \times H_{0}$. In the following lemma, we prove, in particular, that the limit in (5.1) exists.

Lemma 5.1. $P \in \widetilde{\Psi}_{b}^{0}\left(H_{0}, E_{0}\right)$ and is a projection (in general not orthogonal) whose image is $\mathcal{H}(\mathcal{D})$. Moreover, the b-principal symbol of $P$ is the orthogonal projection onto the eigenspace of the negative eigenvalues of the b-principal symbol of $D_{0}$.

Proof. We prove this lemma in three steps.

Step I: We first prove that $P \in \widetilde{\Psi}_{b}^{0}\left(H_{0}, E_{0}\right)$. To do so, we work on a neighborhood $[-1,1]_{u} \times H_{0}$ near $H_{0}$ in $\widetilde{X}$ where, see $(4.10)$,

$$
\widetilde{\mathcal{D}}=G_{u}\left(\partial_{u}+D_{u}\right)
$$

where $G_{u}: E_{0} \rightarrow F_{0}$ is unitary and $D_{u} \in \operatorname{Diff}_{b}^{1}\left(H_{0}, E_{0}\right)$ is a Dirac type operator on $H_{0}$, and where both $G_{u}$ and $D_{u}$ are smooth up to $u=0$ and restricting there to define a unitary map $G_{0}: E_{0} \rightarrow F_{0}$ and a formally self-adjoint Dirac type operator $D_{0} \in \operatorname{Diff}_{b}^{1}\left(H_{0}, E_{0}\right)$. By Theorem 2.3 , we know that $\widetilde{\mathcal{D}}^{-1} \in \widetilde{\Psi}_{b}^{-1}(\widetilde{X}, \widetilde{F}, \widetilde{E})$, and the structure of the Schwartz kernel of $\widetilde{\mathcal{D}}^{-1}$ is described in Section 2. To prove that $P \in \widetilde{\Psi}_{b}^{0}\left(H_{0}, E_{0}\right)$, we shall analyze the limit defining $P$ when $\widetilde{\mathcal{D}}^{-1}$ is described in coordinate patches so that its Schwartz kernel takes the form (2.9), and when its Schwartz kernel takes the form (2.6).

Consider first the case (2.9). Let $\mathscr{U}=[0,1]_{v}^{\ell} \times \mathscr{V}$ and $\mathscr{U}^{\prime}=[0,1]_{v}^{\ell} \times \mathscr{V}^{\prime}$ be coordinate patches on $H_{0}$, where $v=\left(v_{1}, \ldots, v_{\ell}\right)$ denotes those boundary defining functions that are common to both coordinate patches $\mathscr{U}$ and $\mathscr{U}^{\prime}$, and where $\mathscr{V} \subset$ $[0, \infty)^{k} \times \mathbb{R}^{n-1-\ell-k}$ and $\mathscr{V}^{\prime} \subset[0, \infty)^{k^{\prime}} \times \mathbb{R}^{n-1-\ell-k^{\prime}}$. Let $y$ denote the coordinates on $\mathscr{V}$ and $y^{\prime}$ the coordinates on $\mathscr{V}^{\prime}$. Then given any open set $\mathscr{W}$ with compact closure in $(-1,1)_{u} \times \mathscr{U}^{\prime}$, for any $\phi \in \dot{C}^{\infty}(\widetilde{X}, \widetilde{F})$ having support in $\mathscr{W}$, according to $(2.9)$, the restriction of $\widetilde{\mathcal{D}}^{-1} \phi$ to $(-1,1)_{u} \times \mathscr{U}$ is of the form

$$
\widetilde{\mathcal{D}}^{-1} \phi=\int_{(-1,1)_{u^{\prime}} \times \mathscr{U}^{\prime}} R\left(v, \frac{v}{v^{\prime}}, u, y, u^{\prime}, y^{\prime}\right) \phi\left(u^{\prime}, v^{\prime}, y^{\prime}\right) d g\left(u^{\prime}, v^{\prime}, y^{\prime}\right),
$$


where $v / v^{\prime}:=\left(v_{1} / v_{1}^{\prime}, \ldots, v_{\ell} / v_{\ell}^{\prime}\right)$ and where $R\left(v, z, u, y, u^{\prime}, y^{\prime}\right)$ satisfies the conditions listed after (2.9). Therefore, in such coordinates, we have

$$
P \varphi=\lim _{\varepsilon \rightarrow 0^{-}}-\gamma_{\varepsilon} \widetilde{\mathcal{D}}^{-1} \gamma_{0}^{*} G_{0} \varphi=-\int_{\mathscr{U}^{\prime}} R\left(v, \frac{v}{v^{\prime}}, 0, y, 0, y^{\prime}\right) G_{0} \varphi\left(v^{\prime}, y^{\prime}\right) d g\left(0, v^{\prime}, y^{\prime}\right),
$$

which represents an element of $\widetilde{\Psi}_{b}^{-\infty}\left(H_{0}, E_{0}\right)$. Consider now the case when the kernel of $\widetilde{\mathcal{D}}^{-1}$ takes the form (2.6). In this case, let $\mathscr{U}=[0,1]_{v}^{\ell} \times \mathbb{R}_{y}^{n-1-\ell}$. For a section $\phi(u, v, y)$ compactly supported in $(-1,1)_{u} \times[0,1]_{v}^{\ell} \times \mathbb{R}_{y}^{n-1-\ell}$, as in Equation (2.4), we can write

$$
\widetilde{\mathcal{D}}^{-1} \phi=\int_{\mathbb{R}^{n}} e^{i u \xi+i y \cdot \eta} v^{i \tau} a(u, v, y, \xi, \tau, \eta) \hat{\phi}(\xi, \tau, \eta) d \xi d \tau d \eta
$$

where $a(u, v, y, \xi, \tau, \eta)$ is the (complete) symbol of $\widetilde{\mathcal{D}}^{-1}$ and with $\hat{\psi}$ denoting the Fourier transform of $\psi$ in $(u, y)$ and the Mellin transform in $v$. Thus, for a compactly supported $\varphi$ on the cross section coordinate patch $[0,1]^{\ell} \times \mathbb{R}^{n-1-\ell}$, we have

$$
P \varphi=\lim _{\varepsilon \rightarrow 0^{-}}-\gamma_{\varepsilon} \widetilde{\mathcal{D}}^{-1}\left(\gamma_{0}^{*} G_{0} \varphi\right)=\int_{\mathbb{R}^{n-1}} v^{i \tau} e^{i y \cdot \eta} b(v, y, \tau, \eta) \hat{\varphi}(\tau, \eta) d \tau d \eta,
$$

where

$$
b(v, y, \tau, \eta):=-\lim _{u \rightarrow 0^{-}} \frac{1}{2 \pi} \int_{\mathbb{R}} e^{i u \xi} a(u, v, y, \xi, \tau, \eta) G_{0} d \xi
$$

provided, of course, that this limit exists. To prove that this limit exists, we note that by definition of the space $\widetilde{\mathcal{D}}^{-1} \in \widetilde{\Psi}_{b}^{-1}(\widetilde{X}, \widetilde{F}, \widetilde{E})$, the symbol $a(u, v, y, \xi, \tau, \eta)$ is a symbol of order -1 with $b$-principal symbol equal to

$$
a_{1}(u, v, y, \xi, \tau, \eta)={ }^{b} \sigma_{1}(\widetilde{\mathcal{D}})(u, v, y, \xi, \tau, \eta)^{-1}=\frac{(-i \xi+\sigma(u, v, y, \tau, \eta)) G_{u}^{-1}}{\xi^{2}+|(\tau, \eta)|^{2}}
$$

where $|\cdot|^{2}=g(\cdot, \cdot)$ and $\sigma$ is the $b$-principal symbol of $D_{u}$. Moreover, it follows from the explicit local parametrix construction of $\widetilde{\mathcal{D}}$ (see e.g. $[16$, Th. 3.33] or [17, Th. $4.11])$ that $a(u, v, y, \xi, \tau, \eta)$ is a rational symbol in $\xi, \tau, \eta$ in the sense that

$$
a(u, v, y, \xi, \tau, \eta) \sim \sum_{j=1}^{\infty} a_{j}(u, v, y, \xi, \tau, \eta)
$$

where

$$
a_{j}(u, v, y, \xi, \tau, \eta)=\frac{p_{j}(u, v, y, \xi, \tau, \eta)}{\left(\xi^{2}+|(\tau, \eta)|^{2}\right)^{j}}
$$

with $p_{j}(u, v, y, \xi, \tau, \eta)$ a polynomial of degree $j$ in $(\xi, \tau, \eta)$. Note that by $(5.3)$ $a_{1}(u, v, y, \xi, \tau, \eta)$ certainly satisfies this property and a straightforward induction argument shows that this holds for each $j$. Thus, we just have to understand

$$
-\lim _{u \rightarrow 0^{-}} \frac{1}{2 \pi} \int_{\mathbb{R}} e^{i u \xi} a_{j}(u, v, y, \xi, \tau, \eta) G_{0} d \xi
$$

for each $j$. To do so, we write $\xi^{2}+|(\tau, \eta)|^{2}=(\xi+i|(\tau, \eta)|)(\xi-i|(\tau, \eta)|)$ and use Cauchy's theorem to obtain

$$
\begin{array}{r}
\frac{1}{2 \pi} \int_{\mathbb{R}} e^{i u \xi} \frac{p_{j}(u, v, y, \xi, \tau, \eta)}{\left(\xi^{2}+|(\tau, \eta)|^{2}\right)^{j}} d \xi=\frac{i}{2 \pi i} \int_{\mathbb{R}} e^{i u \xi} \frac{p_{j}(u, v, y, \xi, \tau, \eta)}{(\xi+i|(\tau, \eta)|)^{j}(\xi-i|(\tau, \eta)|)^{j}} d \xi \\
=\left.\frac{-i}{(j-1) !}\left(\frac{d}{d \xi}\right)^{j-1}\right|_{\xi=-i|(\tau, \xi)|}\left(e^{i u \xi} \frac{p_{j}(u, v, y, \xi, \tau, \eta)}{(\xi-i|(\tau, \eta)|)^{j}}\right) .
\end{array}
$$


Here, recalling that $u<0$, we shifted the line $\mathbb{R}=\{\Im \xi=0\}$ down to $\{\Im \xi=-\infty\}$ where the integral vanishes, and we picked up a pole at $\xi=-i|(\tau, \eta)|$. Therefore,

$$
\begin{aligned}
-\lim _{u \rightarrow 0^{-}} \frac{1}{2 \pi} \int_{\mathbb{R}} e^{i u \xi} a_{j}(u, v, y, \xi, \tau, \eta) G_{0} d \xi & \\
= & \left.\frac{i}{(j-1) !}\left(\frac{d}{d \xi}\right)^{j-1}\right|_{\xi=-i|(\tau, \xi)|}\left(\frac{p_{j}(u, v, y, \xi, \tau, \eta)}{(\xi-i|(\tau, \eta)|)^{j}}\right) G_{0} .
\end{aligned}
$$

It's easily checked that the right-hand side is homogeneous of degree $1-j$ for each $j=1,2,3, \ldots$. It follows that the symbol $b(v, y, \tau, \eta)$ in (5.2) is a classical symbol of order 0 in $(\tau, \eta)$ and using (5.3), the $b$-principal symbol of $b(v, y, \tau, \eta)$ is equal to

$$
\begin{aligned}
\frac{p_{1}(0, v, y,-i|(\tau, \eta)|, \tau, \eta)}{-2|(\tau, \eta)|} G_{0} & =\frac{(|(\tau, \eta)|-\sigma(0, v, y, \tau, \eta))}{2|(\tau, \eta)|} \\
& =\frac{1}{2}\left(\operatorname{Id}-\frac{\sigma(0, v, y, \tau, \eta)}{|(\tau, \eta)|}\right) .
\end{aligned}
$$

In particular, since $\sigma(0, v, y, \tau, \eta)^{2}=|(\tau, \eta)|^{2}$, the leading order part of $b(v, y, \tau, \eta)$ is the orthogonal projection onto the eigenspace of the negative eigenvalues of the $b$-principal symbol of $D_{0}$. Finally, it is straightforward to check that, from (5.2), a lacunary condition on $a(u, v, y, \xi, \tau, \eta)$ implies a lacunary condition on $b(v, y, \tau, \eta)$. In conclusion, we have shown that $P \in \widetilde{\Psi}_{b}^{0}\left(H_{0}, E_{0}\right)$.

Step II: We now prove that $\operatorname{ran} P=\mathcal{H}(\mathcal{D})$. The proof of this step is similar to Seeley [30, Th. 5]. We first show that $P=\operatorname{Id}$ on $\mathcal{H}(\mathcal{D})$. Let $\varphi=\gamma_{0^{-}} \phi$, where $\gamma_{0^{-}}=\lim _{\varepsilon \rightarrow 0^{-}} \gamma_{\varepsilon}, \phi \in H_{b}^{\infty}(X, E)$, and $\mathcal{D} \phi=0$, and define

$$
\widetilde{\phi}:= \begin{cases}\phi & \text { on } X \\ 0 & \text { on } \widetilde{X} \backslash X .\end{cases}
$$

Since $\mathcal{D} \phi=0$ and, according to (4.10), $\widetilde{\mathcal{D}}=G_{u}\left(\partial_{u}+D_{u}\right)$ near $H_{0}$, and the derivative of the Heaviside function is the delta distribution, it follows that

$$
\widetilde{\mathcal{D}} \widetilde{\phi}=-\delta_{0} \otimes G_{0} \varphi=-\gamma_{0}^{*} G_{0} \varphi,
$$

since $\gamma_{0}^{*}=\delta_{0} \otimes$. with $\delta_{0}$ the delta distribution concentrated at the hypersurface $\{0\} \times H_{0}$. Thus, $\widetilde{\phi}=-\widetilde{\mathcal{D}}^{-1} \gamma_{0}^{*} G \varphi$, and so

$$
P \varphi:=-\gamma_{0^{-}}\left(\widetilde{\mathcal{D}}^{-1} \gamma_{0}^{*} G \varphi\right)=\gamma_{0^{-}}(\widetilde{\phi})=\gamma_{0^{-}}(\phi)=\varphi .
$$

Hence, $P=\operatorname{Id}$ on $\mathcal{H}(\mathcal{D})$. We now show that $P^{2}=P$. Let $\varphi \in H_{b}^{\infty}\left(H_{0}, E_{0}\right)$. Then by definition of $P$, we have

$$
P \varphi=\gamma_{0^{-}} \phi=\varphi,
$$

where $\phi:=-\left.\left(\widetilde{\mathcal{D}}^{-1} \gamma_{0}^{*} G \varphi\right)\right|_{X}$. Note that our analysis in Step I shows that $\phi \in$ $H_{b}^{\infty}(X, E)$. Also, we clearly have $\mathcal{D} \phi=0$. Thus, $\gamma_{0^{-}} \phi \in \mathcal{H}(\mathcal{D})$, so as we know that $P=\operatorname{Id}$ on $\mathcal{H}(\mathcal{D})$, it follows that

$$
P^{2} \varphi=P(P \varphi)=P\left(\gamma_{0^{-}} \phi\right)=\gamma_{0^{-}} \phi=P \varphi .
$$

Our proof is now complete.

We now define

$$
\mathcal{C}:=P P^{*}\left[P P^{*}+\left(\operatorname{Id}-P^{*}\right)(\operatorname{Id}-P)\right]^{-1} .
$$


Then, the right side is the orthogonal projection onto ran $P$ by Lemma 12.8 of [5] (see also [2]). Now by Lemma 5.1 and (5.4), we can see that the $b$-principal symbol of $\mathcal{C}$ is also the orthogonal projection onto the eigenspace of the negative eigenvalues of the $b$-principal symbol of $D_{0}$. To complete the proof of Theorem 1.1, it remains to show that $\mathcal{C} \in \widetilde{\Psi}_{b}^{0}\left(H_{0}, E_{0}\right)$. To show this, let

$$
A=P P^{*}+\left(\mathrm{Id}-P^{*}\right)(\mathrm{Id}-P) .
$$

By Lemma 5.1, the $b$-principal symbol of $P$ is also a projection, so that

$$
{ }^{b} \sigma_{0}(A)={ }^{b} \sigma_{0}(P){ }^{b} \sigma_{0}(P)^{*}+\left(\operatorname{Id}-{ }^{b} \sigma_{0}(P)\right)\left(\operatorname{Id}-{ }^{b} \sigma_{0}(P)^{*}\right)
$$

is always invertible, which means that $A$ is $b$-elliptic. Moreover, by (2.11) any normal operator of $A$ equals

$$
N(A)(\tau)=N(P)(\tau) N(P)(\tau)^{*}+\left(\operatorname{Id}-N(P)(\tau)^{*}\right)(\operatorname{Id}-N(P)(\tau)),
$$

and $N(P)(\tau)$ is a projection since $N(P)(\tau)^{2}=N\left(P^{2}\right)(\tau)=N(P)(\tau)$. Thus, we can see that $N(A)(\tau)$ is invertible for all real $\tau$. Thus, by Theorem 2.1 and 2.3, it follows that $A^{-1} \in \widetilde{\Psi}_{b}^{0}\left(H_{0}, E_{0}\right)$. Finally, by the composition properties of $b$ pseudodifferential operator, we conclude that $\mathcal{C}=P P^{*} A^{-1}$ is also in $\widetilde{\Psi}_{b}^{0}\left(H_{0}, E_{0}\right)$. This completes the proof of Theorem 1.1.

5.2. Proof of Theorem 1.2. We now assume that $E=F$ and $\mathcal{D}$ is formally self-adjoint. This, in particular, implies that $G_{0}^{2}=-\mathrm{Id}$ and $G_{0}^{*}=-G_{0}$. Recall that

$$
\Omega(\varphi, \psi):=\left\langle G_{0} \varphi, \psi\right\rangle_{0} \text { for } \varphi, \psi \in L_{b}^{2}\left(H_{0}, E_{0}\right)
$$

is a Hermitian symplectic form on $L_{b}^{2}\left(H_{0}, E_{0}\right)$ where $\langle,\rangle_{0}$ is the $L_{b}^{2}$ inner product on $H_{0}$. We begin with the following lemma.

Lemma 5.2. The image $\operatorname{ran} \mathcal{P}$ of an orthogonal projector $\mathcal{P}$ on $L_{b}^{2}\left(H_{0}, E_{0}\right)$ is Lagrangian with respect to $\Omega$ if and only if

$$
G_{0} \mathcal{P}=(\mathrm{Id}-\mathcal{P}) G_{0}
$$

Proof. By definition, the subspace $\operatorname{ran} \mathcal{P} \subset L_{b}^{2}\left(H_{0}, E_{0}\right)$ is Lagrangian with respect to $\Omega$ means that $\operatorname{ran} \mathcal{P}=(\operatorname{ran} \mathcal{P}) \frac{\perp}{\Omega}$ where

$$
(\operatorname{ran} \mathcal{P})_{\Omega}^{\perp}=\left\{\varphi \in L_{b}^{2}\left(H_{0}, E_{0}\right) \mid \Omega(\varphi, \psi)=\left\langle G_{0} \varphi, \psi\right\rangle_{0}=0 \quad \forall \psi \in \operatorname{ran} \mathcal{P}\right\} .
$$

Therefore, the Lagrangian property of $\operatorname{ran} \mathcal{P}$ is equivalent to

$$
G_{0}: \operatorname{ran} \mathcal{P} \rightarrow(\operatorname{ran} \mathcal{P})^{\perp}=\operatorname{ran}(\operatorname{Id}-\mathcal{P}) \quad \text { is an isomorphism. }
$$

Now if $G_{0} \mathcal{P}=(\operatorname{Id}-\mathcal{P}) G_{0}$, then certainly (5.6) holds. Conversely, if (5.6) holds, then it follows that $G_{0}^{*}=-G_{0}: \operatorname{ran}(\operatorname{Id}-\mathcal{P}) \rightarrow \operatorname{ran} \mathcal{P}$, which implies that $G_{0}:$ $\operatorname{ran}(\mathrm{Id}-\mathcal{P}) \rightarrow \operatorname{ran} \mathcal{P}$. Hence,

$$
\begin{aligned}
G_{0} \mathcal{P}=(\operatorname{Id}-\mathcal{P}) G_{0} \mathcal{P} & =(\operatorname{Id}-\mathcal{P}) G_{0} \mathcal{P}+0 \\
& =(\operatorname{Id}-\mathcal{P}) G_{0} \mathcal{P}+(\operatorname{Id}-\mathcal{P}) G_{0}(\mathrm{Id}-\mathcal{P}) \\
& =(\operatorname{Id}-\mathcal{P}) G_{0}(\mathcal{P}+\operatorname{Id}-\mathcal{P})=(\operatorname{Id}-\mathcal{P}) G_{0}
\end{aligned}
$$

Thus, $G_{0} \mathcal{P}=(\operatorname{Id}-\mathcal{P}) G_{0}$ and our proof is now complete. 
Thus, according to this lemma, to show that the closure of $\mathcal{H}(\mathcal{D})$ in $L_{b}^{2}\left(H_{0}, E_{0}\right)$, which for simplicity we again denote by $\mathcal{H}(\mathcal{D})$, is a Lagrangian subspace with respect to $\Omega$, it is sufficient to show that

$$
G_{0} \mathcal{C}=(\operatorname{Id}-\mathcal{C}) G_{0}, \quad \text { equivalently } \quad \mathcal{C} G_{0}=G_{0}(\operatorname{Id}-\mathcal{C})
$$

since $\operatorname{ran}(\mathcal{C})=\mathcal{H}(\mathcal{D})$. To do so, we need the following extension of Grubb's result $[10$, Th. 7.5$]$ to the category of manifolds with multi-cylindrical end boundaries.

Lemma 5.3. We have

$$
P^{*} G_{0}=G_{0}(\mathrm{Id}-P) .
$$

Proof. First, we claim that for any $\phi \in H_{b}^{\infty}(X, E)$, we have

$$
\phi=\left(r \widetilde{\mathcal{D}}^{-1} e\right) \mathcal{D} \phi-\left(r \widetilde{\mathcal{D}}^{-1} e\right) \gamma_{0}^{*} G_{0} \gamma_{0} \phi
$$

where $r$ is the restriction map from $\tilde{X}$ to $X, e$ is the extension map by 0 from $X$ to $\widetilde{X}$. This identity can be found in Booß-Bavnbek and Wojciechowski's book [5, Lem. 12.7] for the smooth closed manifold case. To prove this, we define

$$
\widetilde{\phi}:= \begin{cases}\phi & \text { on } X \\ 0 & \text { on } \widetilde{X} \backslash X .\end{cases}
$$

Since $\widetilde{\mathcal{D}}=G_{u}\left(\partial_{u}+D_{u}\right)$ near $H_{0}$ as described in (4.10), and the derivative of the Heaviside function is the delta distribution, it follows that

$$
r \widetilde{\mathcal{D}} \widetilde{\phi}=\mathcal{D} \phi-\gamma_{0}^{*} G_{0} \gamma_{0} \phi
$$

Multiplying both sides by $r \widetilde{\mathcal{D}}^{-1} e$ we get (5.8). In particular, applying $\gamma_{0}$ to both sides of (5.8), we obtain

$$
P \gamma_{0} \phi=\gamma_{0} \phi-\gamma_{0} \widetilde{\mathcal{D}}^{-1} e \mathcal{D} \phi
$$

Second, we follow the proof of Theorem 7.5 in [10]. Let us denote $L^{2}$-pairings over $X\left(H_{0}\right)$ by angular brackets $\langle,\rangle_{X}\left(\langle,\rangle_{0}\right)$ and distributional pairings by parentheses. Then, for $\phi \in H_{b}^{\infty}(X, E)$ and $\psi \in H_{b}^{\infty}\left(H_{0}, E_{0}\right)$, by (5.9) we have

$$
\left\langle\gamma_{0} \widetilde{\mathcal{D}}^{-1} e \mathcal{D} \phi, G_{0} \psi\right\rangle_{0}=\left\langle\left(\gamma_{0} \phi-P \gamma_{0} \phi\right), G_{0} \psi\right\rangle_{0}=\left\langle(\mathrm{Id}-P) \gamma_{0} \phi, G_{0} \psi\right\rangle_{0} .
$$

As distributions we can write the left-hand side of (5.10) as

$$
\begin{aligned}
\left\langle\gamma_{0} \widetilde{\mathcal{D}}^{-1} e \mathcal{D} \phi, G_{0} \psi\right\rangle_{0} & =\left(\gamma_{0}^{*} G_{0} \psi\right)\left(\widetilde{\mathcal{D}}^{-1} e \mathcal{D} \phi\right) \\
& =\left(r \widetilde{\mathcal{D}}^{-1} \gamma_{0}^{*} G_{0} \psi\right)(\mathcal{D} \phi) \\
& =\left\langle\mathcal{D} \phi, r \widetilde{\mathcal{D}}^{-1} \gamma_{0}^{*} G_{0} \psi\right\rangle_{X} \\
& =\left\langle\mathcal{D} \phi, r \widetilde{\mathcal{D}}^{-1} \gamma_{0}^{*} G_{0} \psi\right\rangle_{X}-\left\langle\phi, \mathcal{D} r \widetilde{\mathcal{D}}^{-1} \gamma_{0}^{*} G_{0} \psi\right\rangle_{X}
\end{aligned}
$$

since $\mathcal{D} r \widetilde{\mathcal{D}}^{-1} \gamma_{0}^{*} G_{0} \psi=0$ over $X$. By Green's formula, the right-hand side equals

$$
\begin{aligned}
\left\langle G_{0} \gamma_{0} \phi, \gamma_{0} \widetilde{\mathcal{D}}^{-1} \gamma_{0}^{*} G_{0} \psi\right\rangle_{0} & =-\left\langle G_{0} \gamma_{0} \phi, P \psi\right\rangle_{0} \\
& =-\left\langle P^{*} G_{0} \gamma_{0} \phi, \psi\right\rangle_{0}=-\left\langle G_{0} P^{*} G_{0} \gamma_{0} \phi, G_{0} \psi\right\rangle_{0} .
\end{aligned}
$$

Equating the far right term with the far right term in (5.10), we obtain $-G_{0} P^{*} G_{0}=$ Id $-P$, which, after multiplication by $G_{0}$, proves our result. 
As before, we put $A=P P^{*}+\left(\operatorname{Id}-P^{*}\right)(\operatorname{Id}-P)$. Then using the identity in (5.7), we obtain

$$
\begin{aligned}
A G_{0} & =P\left(P^{*} G_{0}\right)+\left(\operatorname{Id}-P^{*}\right)\left((\operatorname{Id}-P) G_{0}\right) \\
& =P\left(G_{0}(\operatorname{Id}-P)\right)+\left(\operatorname{Id}-P^{*}\right)\left(G_{0} P^{*}\right) \\
& =\left(P G_{0}\right)(\operatorname{Id}-P)+\left(\left(\operatorname{Id}-P^{*}\right) G_{0}\right) P^{*} \\
& =\left(G_{0}\left(\mathrm{Id}-P^{*}\right)\right)(\operatorname{Id}-P)+\left(G_{0} P\right) P^{*} \\
& =G_{0}\left(\left(\mathrm{Id}-P^{*}\right)(\operatorname{Id}-P)+P P^{*}\right)=G_{0} A .
\end{aligned}
$$

Thus, $A G_{0}=G_{0} A$. Hence, $G_{0} A^{-1}=A^{-1} G_{0}$. Again using (5.7), we see that

$$
\begin{aligned}
\mathcal{C} G_{0}=P P^{*} A^{-1} G_{0}=P P^{*} G_{0} A^{-1} & =P G_{0}(\mathrm{Id}-P) A^{-1} \\
& =G_{0}\left(\mathrm{Id}-P^{*}\right)(\mathrm{Id}-P) A^{-1} .
\end{aligned}
$$

Now

$$
\begin{aligned}
\left(\mathrm{Id}-P^{*}\right)(\mathrm{Id}-P) A^{-1} & =\left(P P^{*}+\left(\mathrm{Id}-P^{*}\right)(\mathrm{Id}-P)\right) A^{-1}-P P^{*} A^{-1} \\
& =A A^{-1}-\mathcal{C}=\mathrm{Id}-\mathcal{C}
\end{aligned}
$$

Combining this and (5.11) proves

$$
\mathcal{C} G_{0}=G_{0}(\mathrm{Id}-\mathcal{C}) .
$$

Hence by Lemma $5.2, \mathcal{H}(\mathcal{D})$ is a Lagrangian subspace with respect to $\Omega$. Now let us write

$$
\mathcal{C}=\frac{1}{2}\left(\begin{array}{ll}
C_{11} & C_{12} \\
C_{21} & C_{22}
\end{array}\right)
$$

with respect to the decomposition $L_{b}^{2}\left(H_{0}, E_{0}\right)=L_{b}^{2}\left(H_{0}, E_{0}^{+}\right) \oplus L_{b}^{2}\left(H_{0}, E_{0}^{-}\right)$. Then using $\mathcal{C}^{*}=\mathcal{C}$ and $\mathcal{C} G_{0}=G_{0}(\mathrm{Id}-\mathcal{C})$ it is easy to check that $C_{11}=\mathrm{Id}, C_{22}=\mathrm{Id}$, $C_{21}: L_{b}^{2}\left(Y, E_{Y}^{+}\right) \rightarrow L_{b}^{2}\left(Y, E_{Y}^{-}\right)$is unitary with $C_{12}=C_{21}^{-1}$. Therefore, setting $\kappa_{0}:=C_{12}$, we conclude that

$$
\mathcal{C}=\frac{1}{2}\left(\begin{array}{cc}
\operatorname{Id} & \kappa_{0}^{-1} \\
\kappa_{0} & \mathrm{Id}
\end{array}\right)
$$

This completes the proof of Theorem 1.2.

5.3. Proof of Theorem 1.3. Assume that $E=F, \mathcal{D}$ is formally self-adjoint, and $\mathcal{D}=G_{0}\left(\partial_{u}+D_{0}\right)$ over $[-1,0]_{u} \times H_{0}$. Let $\widetilde{E}:=E \sqcup_{G_{0}} E$ be the doubled vector bundle over the doubled manifold $\widetilde{X}=X \sqcup_{H_{0}}(-X)$ constructed in Subsection 4.2 and let $\widetilde{\mathcal{D}}:=(\mathcal{D},-\mathcal{D})$ be the corresponding invertible double. We define

$$
P_{ \pm}:= \pm \lim _{\varepsilon \rightarrow 0^{ \pm}} \gamma_{\varepsilon} \widetilde{\mathcal{D}}^{-1} \gamma_{0}^{*} G_{0}: H_{b}^{\infty}\left(H_{0}, E_{0}\right) \rightarrow H_{b}^{\infty}\left(H_{0}, E_{0}\right)
$$

To be precise, $P_{ \pm}$really defines a map on $H_{b}^{\infty}\left(H_{0},\left.\widetilde{E}\right|_{H_{0}}\right)$ but we make the following 'left' identification of $E_{0}=\left.E\right|_{H_{0}}$ with $\left.\widetilde{E}\right|_{H_{0}}$ :

$$
\left.E_{0} \longleftrightarrow \widetilde{E}\right|_{H_{0}}:=\left.E_{0} \sqcup_{G_{0}} E_{0} \Longleftrightarrow E_{0} \ni \varphi \longleftrightarrow\left[\left(\varphi, G_{0} \varphi\right)\right] \in \widetilde{E}\right|_{H_{0}} .
$$

As a result of this 'left' identification, given any $\phi \in H_{b}^{\infty}(X, E)$ with $\left.\phi\right|_{H_{0}}=\varphi \in$ $H_{b}^{\infty}\left(H_{0}, E_{0}\right)$, considering $\phi$ as sections of $\widetilde{E}$ over $X$ and $-X$, we have

$$
\left.\phi \in H_{b}^{\infty}(X, \widetilde{E}) \Longrightarrow \phi\right|_{H_{0}}=\varphi,\left.\quad \phi \in H_{b}^{\infty}(-X, \widetilde{E}) \Longrightarrow \phi\right|_{H_{0}}=G_{0} \varphi
$$


the latter equality holds because restriction of $\phi$ from $X$ equals $\varphi$ on the left factor of $E_{0} \sqcup_{G_{0}} E_{0}$, which is equivalent to $G_{0} \varphi$ on the right factor of $E_{0} \sqcup_{G_{0}} E_{0}$. The 'left' identification is important because then the Cauchy data space of $\widetilde{\mathcal{D}}$ taken from the left manifold $X$ of $\widetilde{X}$ is exactly the same as the Cauchy data space of the original operator $\mathcal{D}$ on $X$.

Lemma 5.4. $P_{-}\left(P_{+}\right)$is a projection whose image is exactly the Cauchy data space of the restriction of $\widetilde{\mathcal{D}}$ to $X(-X)$, and

$$
P_{-}+P_{+}=\mathrm{Id}
$$

Proof. In Lemma 5.1 we showed that $P_{-}$is a projection whose image is exactly the Cauchy data space of the restriction of $\widetilde{\mathcal{D}}$ to $X$ and a similar proof establishes the corresponding claim for $P_{+}$.

The proof of (5.13) is similar to Seeley's proof [30, Th. 5] but now in the " $b$ category". Let $\phi \in H_{b}^{\infty}\left([-1,1]_{u} \times H_{0}, \widetilde{E}\right)$ have compact support in $(-1,1)_{u} \times H_{0}$ and let $\psi \in H_{b}^{\infty}\left(H_{0}, E_{0}\right)$. Let us denote distributional pairing by parentheses and, as usual, $L^{2}$-pairings over $H_{0}$ by angular brackets $\langle,\rangle_{0}$. If $\widetilde{\mathcal{K}}:=\widetilde{\mathcal{D}}^{-1} \gamma_{0}^{*} G_{0}$, then

$$
\begin{aligned}
\left\langle\gamma_{0} \phi, G_{0} \psi\right\rangle_{0}=\left(\gamma_{0}^{*} G_{0} \psi\right)(\phi)=(\widetilde{\mathcal{D}} \widetilde{\mathcal{K}} \psi)(\phi) & =(\widetilde{\mathcal{K}} \psi)(\widetilde{\mathcal{D}} \phi) \\
& =\int_{-1}^{1}\langle\widetilde{\mathcal{D}} \phi, \widetilde{\mathcal{K}} \psi\rangle_{0} d u,
\end{aligned}
$$

where we used the fact that from the proof of Lemma 5.1, $\widetilde{\mathcal{K}} \psi=\widetilde{\mathcal{D}}^{-1} \gamma_{0}^{*} G_{0} \psi$ has a left-hand limit at $H_{0}$ and a similar proof shows that it has a right-hand limit at $H_{0}$, so the function $\widetilde{\mathcal{K}} \psi$ is in $H_{b}^{\infty}$ off $H_{0}$ with at most a jump discontinuity at $H_{0}$. In particular, we can write

$$
\int_{-1}^{1}\langle\widetilde{\mathcal{D}} \phi, \widetilde{\mathcal{K}} \psi\rangle_{0} d u=\lim _{r \rightarrow 0^{+}} \int_{|u|>r}\langle\widetilde{\mathcal{D}} \phi, \widetilde{\mathcal{K}} \psi\rangle_{0} d u
$$

By (4.10) we know that $\widetilde{\mathcal{D}}=G_{0}\left(\partial_{u}+D_{u}\right)$ over $[-1,1]_{u} \times H_{0}$, so we can evaluate the right-hand integral as follows:

$$
\begin{aligned}
\int_{|u|>r}\langle\widetilde{\mathcal{D}} \phi, \widetilde{\mathcal{K}} \psi\rangle_{0} d u & =\int_{|u|>r}\left\langle\left(G_{0}\left(\partial_{u}+D_{u}\right)\right) \phi, \widetilde{\mathcal{K}} \psi\right\rangle_{0} d u \\
& =-\int_{|u|>r}\left\langle\partial_{u} \phi, G_{0} \widetilde{\mathcal{K}} \psi\right\rangle_{0} d u+\int_{|u|>r}\left\langle\phi,\left(G_{0} D_{u}\right) \widetilde{\mathcal{K}} \psi\right\rangle_{0} d u \\
& =-\int_{|u|>r} \partial_{u}\left\langle\phi, G_{0} \widetilde{\mathcal{K}} \psi\right\rangle_{0} d u+\int_{|u|>r}\langle\phi, \widetilde{\mathcal{D}} \widetilde{\mathcal{K}} \psi\rangle_{0} d u \\
& =-\left\langle\gamma_{-r} \phi, G_{0} \gamma_{-r} \widetilde{\mathcal{K}} \psi\right\rangle_{0}+\left\langle\gamma_{r} \phi, G_{0} \gamma_{r} \widetilde{\mathcal{K}} \psi\right\rangle_{0},
\end{aligned}
$$

where we used that $\widetilde{\mathcal{D}} \widetilde{\mathcal{K}}=0$ off $H_{0}$. Taking $r \rightarrow 0^{+}$in (5.15) and equating this with $\left\langle\gamma_{0} \phi, G_{0} \psi\right\rangle_{0}$ in (5.14), and using that $P_{-}:=-\gamma_{0_{-}} \widetilde{\mathcal{K}} \psi$ and $P_{+}:=\gamma_{0+} \widetilde{\mathcal{K}} \psi$, we conclude that

$$
\left\langle\gamma_{0} \phi, G_{0} \psi\right\rangle_{0}=\left\langle\gamma_{0} \phi, G_{0} P_{-} \psi\right\rangle_{0}+\left\langle\gamma_{0} \phi, G_{0} P_{+} \psi\right\rangle_{0}
$$

Since $\phi$ and $\psi$ were arbitrary, it follows that $\mathrm{Id}=P_{-}+P_{+}$, and our proof is complete.

Now to prove Theorem 1.3, we need to demonstrate that $P_{-}=\mathcal{C}$; which reduces to proving that $P_{-}$is orthogonal. To this end, we first prove the following 
Lemma 5.5. We have $G_{0} P_{-}=P_{+} G_{0}$.

Proof. We shall prove that $G_{0} P_{-}=P_{+} G_{0}$ first over ran $P_{-}$and then over $\operatorname{ran}(\mathrm{Id}-$ $\left.P_{-}\right)=\operatorname{ran} P_{+}$.

Let $\varphi \in \operatorname{ran} P_{-} \subset H_{b}^{\infty}\left(H_{0}, E_{0}\right)$. Then $P_{-} \varphi=\varphi$ and there is a $\phi_{1} \in C^{\infty}(X, E)$ such that $\mathcal{D} \phi_{1}=0$ and $\left.\phi_{1}\right|_{H_{0}}=\varphi$. Let $\phi_{2} \in C^{\infty}(-X, E)$ be the same section $\phi_{1}$, but considered on the reversed manifold $-X$. Since $\mathcal{D} \phi_{1}=0$, we have $\widetilde{\mathcal{D}} \phi_{2}=-\mathcal{D} \phi_{2}=0$, therefore $\left.\phi_{2}\right|_{H_{0}} \in \operatorname{ran} P_{+}$or $P_{+}\left(\left.\phi_{2}\right|_{H_{0}}\right)=\left.\phi_{2}\right|_{H_{0}}$. In view of the identification (5.12), we have $\left.\phi_{2}\right|_{H_{0}}=G_{0} \varphi$, therefore

$$
G_{0}\left(P_{-} \varphi\right)=G_{0}(\varphi)=\left.\phi_{2}\right|_{H_{0}}=P_{+}\left(\left.\phi_{2}\right|_{H_{0}}\right)=P_{+}\left(G_{0} \varphi\right)=P_{+} G_{0} \varphi .
$$

Now let $\varphi \in \operatorname{ran}\left(\operatorname{Id}-P_{-}\right)=\operatorname{ran} P_{+} \subset H_{b}^{\infty}\left(H_{0}, E_{0}\right)$. Then $P_{-} \varphi=0$, so we just have to prove that $P_{+} G_{0} \varphi=0$ too. Since $\varphi \in \operatorname{ran} P_{+}$, we know that $P_{+} \varphi=\varphi$ and there is a $\phi_{2} \in C^{\infty}(-X, E)$ such that $\mathcal{D} \phi_{2}=0$ and $\left.\phi_{2}\right|_{H_{0}}=\varphi$. Let $\phi_{1} \in C^{\infty}(X, E)$ be the same section $\phi_{2}$, but considered on the left manifold $X$. Since $\mathcal{D} \phi_{2}=0$, we have $\widetilde{\mathcal{D}} \phi_{1}=\mathcal{D} \phi_{1}=0$, therefore $\left.\phi_{1}\right|_{H_{0}} \in \operatorname{ran} P_{-}$or $P_{-}\left(\left.\phi_{1}\right|_{H_{0}}\right)=\left.\phi_{1}\right|_{H_{0}}$. Because $\left.\phi_{2}\right|_{H_{0}}=\varphi$ from the right manifold $-X$, in view of the identification (5.12), from the left we must have $\left.\phi_{1}\right|_{H_{0}}=-G_{0} \varphi$. Hence, $P_{-}\left(-G_{0} \varphi\right)=-G_{0} \varphi$, and so

$$
P_{+} G_{0} \varphi=P_{+} P_{-}\left(G_{0} \varphi\right)=\left(\operatorname{Id}-P_{-}\right) P_{-}\left(G_{0} \varphi\right)=0 .
$$

Now combining the identity (5.13) with Lemma 5.5, we obtain

$$
G_{0} P_{-}=\left(\mathrm{Id}-P_{-}\right) G_{0} .
$$

On the other hand, we also know that $G_{0} P_{-}^{*}=\left(\mathrm{Id}-P_{-}\right) G_{0}$ from (5.7). Hence, $P_{-}=P_{-}^{*}$ and our proof of Theorem 1.3 is now complete.

\section{Relative indeX Formulæ AND the BoJARSki CONJECTURE}

In this section we prove Theorems 1.4 and 1.5 .

6.1. Proof of Theorem 1.4. The proof of Theorem 1.4 consists of three steps.

Step I: First, we consider the following general abstract situation. Let $V_{0}, V_{1}, V_{2}$ be topological vector spaces and let

$$
T: V_{1} \rightarrow V_{2} \quad, \quad \gamma_{0}: V_{1} \rightarrow V_{0}
$$

be continuous surjective linear transformations. Suppose there is a projection

$$
\mathcal{C}: V_{0} \rightarrow V_{0}
$$

whose image is the generalized Cauchy data space of $T$ :

$$
\mathcal{H}(T):=\gamma_{0} \operatorname{ker} T=\left\{\gamma_{0} \phi \mid \phi \in V_{1}, T \phi=0\right\} \subset V_{0}
$$

and the generalized unique continuation property holds:

$$
\psi \in \operatorname{ran} \mathcal{C} \Longleftrightarrow \exists ! \phi \in V_{1}, T \phi=0 \text { and } \gamma_{0} \phi=\psi .
$$

Given a projection $\mathcal{P}: V_{0} \rightarrow V_{0}$, consider the linear map

$$
T_{\mathcal{P}}: \operatorname{dom}\left(T_{\mathcal{P}}\right) \rightarrow V_{2}
$$

where

$$
\operatorname{dom}\left(T_{\mathcal{P}}\right):=\left\{\phi \in V_{1} \mid \mathcal{P}\left(\gamma_{0} \phi\right)=0\right\} \subset V_{1} .
$$


Proposition 6.1. For an arbitrary projection $\mathcal{P}: V_{0} \rightarrow V_{0}$, the operator

$$
T_{\mathcal{P}}: \operatorname{dom}\left(T_{\mathcal{P}}\right) \rightarrow V_{2}
$$

is Fredholm; that is, has a finite-dimensional kernel and cokernel, if and only if

$$
\mathcal{P C}: \operatorname{ran} \mathcal{C} \rightarrow \operatorname{ran} \mathcal{P}
$$

is Fredholm, in which case the following index formula holds:

$$
\operatorname{ind} T_{\mathcal{P}}=\operatorname{ind}(\mathcal{P}, \mathcal{C}):=\operatorname{ind}(\mathcal{P C}: \operatorname{ran} \mathcal{C} \rightarrow \operatorname{ran} \mathcal{P}) .
$$

Proof. We just need to establish isomorphisms between kernels: $\operatorname{ker} T_{\mathcal{P}} \cong \operatorname{ker} \mathcal{P C}$ and cokernels: $\operatorname{coker} T_{\mathcal{P}}:=V_{2} / \operatorname{ran} T_{\mathcal{P}} \cong \operatorname{ran} \mathcal{P} / \operatorname{ran} \mathcal{P C}=:$ coker $\mathcal{P C}$. This shows that $T_{\mathcal{P}}$ is Fredholm if and only if $\mathcal{P C}$ is Fredholm. The first congruence is easy: Using that

$$
\psi \in \operatorname{ran} \mathcal{C} \quad \Longleftrightarrow \quad \exists ! \phi \in V_{1}, T \phi=0 \text { and } \gamma_{0} \phi=\psi
$$

it follows that

$$
\begin{aligned}
\psi \in \operatorname{ran} \mathcal{C}, \mathcal{P} \psi=0 & \Longleftrightarrow \exists ! \phi, T \phi=0 \text { and } \gamma_{0} \phi=\psi \text { and } \mathcal{P} \psi=0 \\
& \Longleftrightarrow \exists ! \phi, T \phi=0 \text { and } \mathcal{P} \gamma_{0} \phi=0 \text { and } \gamma_{0} \phi=\psi \\
& \Longleftrightarrow \exists ! \phi \in \operatorname{ker} T_{\mathcal{P}} \text { with } \gamma_{0} \phi=\psi
\end{aligned}
$$

Therefore the map

$$
\operatorname{ker} T_{\mathcal{P}} \ni \phi \mapsto \gamma_{0} \phi \in \operatorname{ker} \mathcal{P C}
$$

is an isomorphism.

It remains to prove that coker $T_{\mathcal{P}} \cong \operatorname{coker} \mathcal{P C}$. To prove this, we define a map

$$
f: V_{0} \rightarrow V_{2} / \operatorname{ran} T_{\mathcal{P}}=\operatorname{coker} T_{\mathcal{P}}
$$

as follows. Let $\psi \in V_{0}$. Then there is a $\phi \in V_{1}$ such that $\gamma_{0} \phi=\psi$. We define

$$
f(\psi):=[T \phi] \in V_{2} / \operatorname{ran} T_{\mathcal{P}},
$$

where [ ] denotes equivalence class. This is well-defined because if $\gamma_{0} \tilde{\phi}=\psi$ also, then $\mathcal{P} \gamma_{0}(\tilde{\phi}-\phi)=\mathcal{P}(0)=0$, so $\tilde{\phi}-\phi \in \operatorname{dom}\left(T_{\mathcal{P}}\right)$, and thus,

$$
[T \tilde{\phi}]=[T \phi+T(\tilde{\phi}-\phi)]=[T \phi] .
$$

Since we know that $T: V_{1} \rightarrow V_{2}$ is surjective, $f$ is also surjective. Observe that if $\mathcal{P} \psi=0$, then with $\phi \in V_{1}$ such that $\gamma_{0} \phi=\psi$, we have $\phi \in \operatorname{dom}\left(T_{\mathcal{P}}\right)$, so $T \phi \in \operatorname{ran} T_{\mathcal{P}}$ and thus $f(\psi)=[T \phi]=0$. Hence,

$$
f: \operatorname{ker} \mathcal{P} \rightarrow \operatorname{ran} T_{\mathcal{P}} .
$$

Therefore $f$ descends to a (still surjective) map on the quotient:

$$
\tilde{f}: V_{0} / \operatorname{ker} \mathcal{P} \rightarrow V_{2} / \operatorname{ran} T_{\mathcal{P}} .
$$

We are given that $\mathcal{P}$ is a projection, so we have a canonical isomorphism

$$
\operatorname{ran} \mathcal{P} \cong V_{0} / \operatorname{ker} \mathcal{P} \text {. }
$$

Indeed, the map $\operatorname{ran} \mathcal{P} \ni \psi \mapsto[\psi] \in V_{0} / \operatorname{ker} \mathcal{P}$ is certainly one-to-one, and it is surjective because given any $\psi \in V_{0}$, we can write

$$
\psi=\mathcal{P} \psi+(\operatorname{Id}-\mathcal{P}) \psi=\mathcal{P} \psi \quad \text { modulo } \quad \text { ker } \mathcal{P},
$$

as $\operatorname{ker} \mathcal{P}=\operatorname{ran}(\operatorname{Id}-\mathcal{P})$ since $\mathcal{P}$ is a projection. Thus, we obtain a surjective map

$$
\tilde{f}: \operatorname{ran} \mathcal{P} \rightarrow V_{2} / \operatorname{ran} T_{\mathcal{P}} .
$$


We claim that $\operatorname{ker} \tilde{f}=\operatorname{ran} \mathcal{P C}$. Once we prove this, it follows that $\tilde{f}$ descends to an isomorphism of vector spaces

$$
\text { coker } \mathcal{P C}:=\operatorname{ran} \mathcal{P} / \operatorname{ran} \mathcal{P C} \cong V_{2} / \operatorname{ran} T_{\mathcal{P}}=: \operatorname{coker} T_{\mathcal{P}}
$$

which completes our proof. We first show that $\operatorname{ker} \tilde{f} \subset \operatorname{ran} \mathcal{P C}$. An element of $\operatorname{ker} \tilde{f}$ is an element $\psi \in \operatorname{ran} \mathcal{P}$ where we choose $\phi \in V_{1}$ such that $\gamma_{0} \phi=\psi$ and $T \phi \in \operatorname{ran} T_{\mathcal{P}}$. The inclusion $T \phi \in \operatorname{ran} T_{\mathcal{P}}$ means that

$$
\exists \tilde{\phi} \in V_{1}, \mathcal{P} \gamma_{0} \tilde{\phi}=0, T \tilde{\phi}=T \phi .
$$

In particular, $T(\phi-\tilde{\phi})=0$, so $\gamma_{0}(\phi-\tilde{\phi})=\psi-\gamma_{0} \tilde{\phi} \in \operatorname{ran} \mathcal{C}$. Thus,

$$
\psi=\mathcal{P} \psi-0=\mathcal{P} \psi-\mathcal{P} \gamma_{0} \tilde{\phi}=\mathcal{P}\left(\psi-\gamma_{0} \tilde{\phi}\right) \in \operatorname{ran} \mathcal{P C} .
$$

The proof that $\operatorname{ran} \mathcal{P C} \subset \operatorname{ker} \tilde{f}$ is similar: An element of $\operatorname{ran} \mathcal{P C}$ is an element $\psi \in \operatorname{ran} \mathcal{P}$ such that $\psi \in \operatorname{ran} \mathcal{C}$ also. The inclusion $\psi \in \operatorname{ran} \mathcal{C}$ means that

$$
\exists ! \phi \in V_{1}, \gamma_{0} \phi=\psi, T \phi=0 .
$$

Choose $\tilde{\phi} \in V_{1}$ with $\gamma_{0} \tilde{\phi}=\psi$. Then $\mathcal{P} \gamma_{0}(\tilde{\phi}-\phi)=\mathcal{P}(\psi-\psi)=0$, so $\tilde{\phi}-\phi \in \operatorname{dom}\left(T_{\mathcal{P}}\right)$ and thus,

$$
\tilde{f}(\psi)=[T \tilde{\phi}]=[T \phi+T(\tilde{\phi}-\phi)]=[T \phi]=[0]=0 .
$$

Step II: Now we apply Proposition 6.1 to

$$
\mathcal{D}: H_{b}^{\infty}(X, E) \rightarrow H_{b}^{\infty}(X, F) \quad, \quad \gamma_{0}: H_{b}^{\infty}\left(H_{0}, E_{0}\right) \rightarrow H_{b}^{\infty}\left(H_{0}, E_{0}\right),
$$

where $\gamma_{0}$ is restriction to $H_{0}$, which is obviously surjective. The surjective property of $\mathcal{D}$ follows from Theorem 1.1. Also, we know that

$$
\mathcal{C}: H_{b}^{\infty}\left(H_{0}, E_{0}\right) \rightarrow H_{b}^{\infty}\left(H_{0}, E_{0}\right)
$$

has image equal to the Cauchy data space of $\mathcal{D}$ :

$$
\mathcal{H}(\mathcal{D}):=\gamma_{0} \operatorname{ker} \mathcal{D}=\left\{\gamma_{0} \phi \mid \phi \in H_{b}^{\infty}(X, E), \mathcal{D} \phi=0\right\} \subset H_{b}^{\infty}\left(H_{0}, E_{0}\right)
$$

and

$$
\psi \in \operatorname{ran} \mathcal{C} \quad \Longleftrightarrow \quad \exists ! \phi \in H_{b}^{\infty}(X, E), \mathcal{D} \phi=0 \text { and } \gamma_{0} \phi=\psi .
$$

The uniqueness here follows from the unique continuation principle, which also holds for our manifold [1, Cor. 1]. Therefore, we have satisfied all the conditions of Proposition 6.1, so we conclude that

Proposition 6.2. For an arbitrary projection $\mathcal{P} \in G r_{\infty}^{*}(\mathcal{D})$, the operator

$$
\mathcal{D}_{\mathcal{P}}: \operatorname{dom}\left(\mathcal{D}_{\mathcal{P}}\right)_{\infty}:=\left\{\phi \in H_{b}^{\infty}(X, E) \mid \mathcal{P}\left(\gamma_{0} \phi\right)=0\right\} \rightarrow H_{b}^{\infty}(X, F)
$$

is Fredholm; that is, $\mathcal{D}_{\mathcal{P}}$ has a finite-dimensional kernel and cokernel if and only if $\mathcal{P C}: \operatorname{ran} \mathcal{C} \rightarrow \operatorname{ran} \mathcal{P}$ is Fredholm.

Remark 6.3. In applications of Proposition 6.1, the surjective condition of $T$ is very crucial as its proof shows. Here is one case where we can clearly see this point. Let us consider the half infinite cylinder $X=[0, \infty) \times Y$ where $Y$ is compact boundaryless manifold and a Dirac type operator

$$
\mathcal{D}=G\left(\partial_{u}+D_{Y}\right): H_{b}^{\infty}(X, E) \rightarrow H_{b}^{\infty}(X, F)
$$

where $D_{Y}$ is a Dirac type operator over $Y$. We assume that $D_{Y}$ is non-invertible. Then it is easy to see that the Calderón projection $\mathcal{C}$ at $\{0\} \times Y$ is the spectral projection $\Pi_{>}$onto the positive eigenspaces of $D_{Y}$. We choose an augmented 
APS spectral projection $P_{\sigma}=\Pi_{>}+\frac{1-\sigma}{2} \Pi_{0}$ where $\sigma$ is an involution over $\operatorname{ker} D_{Y}$ such that $G \sigma=-\sigma G$ and $\Pi_{0}$ is the orthogonal projection onto ker $D_{Y}$. Then $P_{\sigma} \Pi_{>}: \operatorname{ran}\left(\Pi_{>}\right) \rightarrow \operatorname{ran}\left(P_{\sigma}\right)$ is certainly a Fredholm operator yet it is well-known, and easy to prove, that $\mathcal{D}_{P_{\sigma}}$ is not Fredholm since $D_{Y}$ is not invertible. Therefore, this example shows that Proposition 6.1 does not hold for this simple case. The reason is that the operator $\mathcal{D}: H_{b}^{\infty}(X, E) \rightarrow H_{b}^{\infty}(X, F)$ is not surjective when $D_{Y}$ is not invertible. For instance, given $\varphi \in \operatorname{ker} D_{Y}$, it is easy to check that $\psi:=\frac{1}{1+u} G \varphi(y) \in H_{b}^{\infty}(X, F)$ is not in the image of $\mathcal{D}$. In order to establish a Fredholm theory for $\mathcal{D}$ we need to 'perturb' it as explained in Section 3 of [18].

Step III: For $\mathcal{P} \in G r_{\infty}^{*}(\mathcal{D})$, let us consider the $L_{b}^{2}$ domain:

$$
\operatorname{dom}\left(\mathcal{D}_{\mathcal{P}}\right):=\left\{\phi \in H_{b}^{1}(X, E) \mid \mathcal{P}\left(\gamma_{0} \phi\right)=0\right\},
$$

where $\gamma_{0}: H_{b}^{1}(X, E) \rightarrow H_{b}^{1 / 2}\left(H_{0}, E_{0}\right)$ is restriction to the boundary, and

$$
\mathcal{D}_{\mathcal{P}}: \operatorname{dom}\left(\mathcal{D}_{\mathcal{P}}\right) \rightarrow L_{b}^{2}(X, F)
$$

is regarded as an unbounded operator on $L_{b}^{2}$. To complete the proof of Theorem 1.4, we shall prove that $\mathcal{D}_{\mathcal{P}}$ in $(6.3)$ is $L_{b}^{2}$-Fredholm and its index is the same as the index of $\mathcal{D}_{\mathcal{P}}$ in (6.1):

$$
\text { ind }\left(\mathcal{D}_{\mathcal{P}}: \operatorname{dom}\left(\mathcal{D}_{\mathcal{P}}\right) \subset H_{b}^{1} \rightarrow L_{b}^{2}\right)=\operatorname{ind}\left(\mathcal{D}_{\mathcal{P}}: \operatorname{dom}\left(\mathcal{D}_{\mathcal{P}}\right)_{\infty} \subset H_{b}^{\infty} \rightarrow H_{b}^{\infty}\right) \text {. }
$$

To establish these properties, we first prove

Lemma 6.4. There is an operator $Q$ having the property that for any $s>1 / 2$, $Q: H_{b}^{s}(X, F) \oplus H_{b}^{s+1 / 2}\left(H_{0}, E_{0}\right) \rightarrow H_{b}^{s+1}(X, E)$ is continuous and

$$
Q \circ\left(\begin{array}{c}
\mathcal{D} \\
\mathcal{P} \gamma_{0}
\end{array}\right)=\operatorname{Id}+\mathcal{S}: H_{b}^{s}(X, E) \rightarrow H_{b}^{s}(X, E),
$$

where $\mathcal{S}: H_{b}^{s}(X, E) \rightarrow H_{b}^{\infty}(X, E)$ is a compact operator. In particular ran $\mathcal{D}_{\mathcal{P}}$ is closed and $\operatorname{ker} \mathcal{D}_{\mathcal{P}} \subset H_{b}^{\infty}(X, E)$.

Proof. We define $Q$ as the $1 \times 2$ matrix

$$
Q:=\left[\left(\operatorname{Id}+\mathcal{K}(\operatorname{Id}-\mathcal{C}) \gamma_{0}\right)\left(r \widetilde{\mathcal{D}}^{-1} e\right), \mathcal{K} \mathcal{P}\right],
$$

where $\widetilde{\mathcal{D}}$ is an invertible extension of $\mathcal{D}, r$ is the restriction map from $\widetilde{X}$ to $X, e$ is the extension map by 0 from $X$ to $\widetilde{X}$, and

$$
\mathcal{K}:=-r \widetilde{\mathcal{D}}^{-1} \gamma_{0}^{*} G_{0}: H_{b}^{s}\left(H_{0}, E_{0}\right) \rightarrow H_{b}^{s+1 / 2}(X, E)
$$

is the Poisson operator of $\mathcal{D}$. Recall that $\operatorname{ran} P=\operatorname{ran} \mathcal{C}$ where $P=\gamma_{0} \mathcal{K}$ by $(5.1)$. (Note that if $\mathcal{D}$ is of product type near $H_{0}$ and $\widetilde{\mathcal{D}}$ is the invertible double, then $P=\mathcal{C}$ by Theorem 1.3 , but $P \neq \mathcal{C}$ in general). By $(5.8)$, we have $\left(r \widetilde{\mathcal{D}}^{-1} e\right) \mathcal{D}=$ Id $-\mathcal{K} \gamma_{0}$, and using this we obtain

$$
\begin{aligned}
Q \circ\left(\begin{array}{c}
\mathcal{D} \\
\mathcal{P} \gamma_{0}
\end{array}\right) & =\left[\left(\mathrm{Id}+\mathcal{K}(\operatorname{Id}-\mathcal{C}) \gamma_{0}\right)\left(r \widetilde{\mathcal{D}}^{-1} e\right), \mathcal{K} \mathcal{P}\right]\left(\begin{array}{c}
\mathcal{D} \\
\mathcal{P} \gamma_{0}
\end{array}\right) \\
& =\left(\mathrm{Id}+\mathcal{K}(\mathrm{Id}-\mathcal{C}) \gamma_{0}\right)\left(r \widetilde{\mathcal{D}}^{-1} e\right) \mathcal{D}+\mathcal{K} \mathcal{P} \gamma_{0} \\
& =\mathrm{Id}-\mathcal{K} \gamma_{0}+\mathcal{K}(\mathrm{Id}-\mathcal{C}) \gamma_{0}-\mathcal{K}(\mathrm{Id}-\mathcal{C}) \gamma_{0} \mathcal{K} \gamma_{0}+\mathcal{K} \mathcal{P} \gamma_{0} \\
& =\mathrm{Id}-\mathcal{K} \gamma_{0}+\mathcal{K} \gamma_{0}-\mathcal{K} \mathcal{C} \gamma_{0}-\mathcal{K}(\mathrm{Id}-\mathcal{C}) P \gamma_{0}+\mathcal{K} \mathcal{P} \gamma_{0} \\
& =\mathrm{Id}-\mathcal{K} \mathcal{C} \gamma_{0}+\mathcal{K} \mathcal{P} \gamma_{0}=\mathrm{Id}+\mathcal{S}
\end{aligned}
$$


where $\mathcal{S}=\mathcal{K}(\mathcal{P}-\mathcal{C}) \gamma_{0}$. By assumption, $\mathcal{P}-\mathcal{C} \in \widetilde{\Psi}^{-\infty}\left(H_{0}, E_{0}\right)$, from which the compactness and regularity of $\mathcal{S}$ follows. Note that the regularity property of $\mathcal{S}$ implies that $\operatorname{ker} \mathcal{D}_{\mathcal{P}} \subset H_{b}^{\infty}(X, E)$.

It remains to prove that $\operatorname{ran} \mathcal{D}_{\mathcal{P}}$ is closed. To do so, let $\left\{\phi_{n}\right\}$ be a sequence in $\operatorname{dom}\left(\mathcal{D}_{\mathcal{P}}\right) \subset H_{b}^{1}(X, E)$ with $\psi_{n}:=\mathcal{D}_{\mathcal{P}} \phi_{n} \rightarrow \psi \in L_{b}^{2}(X, F)$. We need to find a $\phi \in \operatorname{dom}\left(\mathcal{D}_{\mathcal{P}}\right)$ with $\mathcal{D}_{\mathcal{P}} \phi=\psi$. To this end, observe that since $\mathcal{S}$ is compact we may assume that $\mathcal{S} \phi_{n}$ converges to an element of $H_{b}^{\infty}(X, E)$. Then applying $\phi_{n}$ to both sides of (6.4) and using that $\phi_{n} \in \operatorname{dom}\left(\mathcal{D}_{\mathcal{P}}\right)$ so that $\mathcal{P} \gamma_{0} \phi_{n}=0$ and that $Q: L_{b}^{2}(X, F) \oplus 0 \rightarrow H_{b}^{1}(X, E)$ is continuous, we see that

$$
\phi_{n}=Q\left(\begin{array}{c}
\psi_{n} \\
0
\end{array}\right)-\mathcal{S} \phi_{n}
$$

converges in the topology of $H_{b}^{1}(X, E)$, say to an element $\phi$. Then

$$
\mathcal{D}_{\mathcal{P}} \phi=\lim _{n \rightarrow \infty} \mathcal{D}_{\mathcal{P}} \phi_{n}=\psi
$$

and, since $\mathcal{P} \gamma_{0} \phi_{n}=0$ for each $n$, we have $\mathcal{P} \gamma_{0} \phi=\lim _{n \rightarrow \infty} \mathcal{P} \gamma_{0} \phi_{n}=0$. This proves that $\operatorname{ran} \mathcal{D}_{\mathcal{P}}$ is closed and completes our proof.

The following proposition completes the proof of Theorem 1.4 except for the relative index formula, which we take care of in a moment.

Proposition 6.5. The Calderón projector of $\mathcal{D}^{*}: C^{\infty}(X, F) \rightarrow C^{\infty}(X, E)$ is the projector $\mathcal{P}^{\prime}:=G_{0}(\mathrm{Id}-\mathcal{C}) G_{0}^{*}$ with $\mathcal{P}^{\prime} \in G r_{\infty}^{*}\left(\mathcal{D}^{*}\right)$, and the $L_{b}^{2}$ adjoint of the map $\mathcal{D}_{\mathcal{P}}$ in $(6.3)$ is the map

$$
\left(\mathcal{D}^{*}\right)_{\mathcal{P}^{\prime}}: \operatorname{dom}\left(\left(\mathcal{D}^{*}\right)_{\mathcal{P}^{\prime}}\right) \rightarrow L_{b}^{2}(X, E)
$$

where

$$
\operatorname{dom}\left(\left(\mathcal{D}^{*}\right)_{\mathcal{P}^{\prime}}\right):=\left\{\phi \in H_{b}^{1}(X, F) \mid \mathcal{P}^{\prime}\left(\gamma_{0} \phi\right)=0\right\} .
$$

Proof. The fact that the Calderón projector of $\mathcal{D}^{*}$ is $G_{0}(\operatorname{Id}-\mathcal{C}) G_{0}^{*}$ follows by applying Theorem 1.2 to the formally self-adjoint Dirac type operator

$$
\left(\begin{array}{cc}
0 & \mathcal{D}^{*} \\
\mathcal{D} & 0
\end{array}\right): C^{\infty}\left(X, E^{\prime}\right) \rightarrow C^{\infty}\left(X, E^{\prime}\right) \quad, \quad E^{\prime}=E \oplus F
$$

To see that the $L_{b}^{2}$ adjoint of the map $\mathcal{D}_{\mathcal{P}}$ is $\left(\mathcal{D}^{*}\right)_{\mathcal{P}^{\prime}}$, let $\varphi \in H_{b}^{\infty}\left(H_{0}, E_{0}\right), f \in$ $H_{b}^{\infty}(X, F)$, and choose $e \in H_{b}^{\infty}(X, E)$ with $\left.e\right|_{H_{0}}=(\operatorname{Id}-\mathcal{P}) \varphi$. Then $e \in \operatorname{dom}\left(\mathcal{D}_{\mathcal{P}}\right)$ and by Green's formula, as a distributional pairing, we see that

$$
\begin{aligned}
\left((\operatorname{Id}-\mathcal{P}) G_{0}^{*} \gamma_{0} f\right)(\varphi)=\left\langle\varphi,(\operatorname{Id}-\mathcal{P}) G_{0}^{*} f\right\rangle_{0} & =\left\langle G_{0}(\operatorname{Id}-\mathcal{P}) \varphi, f\right\rangle_{0} \\
& =\langle\mathcal{D} e, f\rangle_{X}-\left\langle e, \mathcal{D}^{*} f\right\rangle_{X}
\end{aligned}
$$

Now let us assume that $f \in \operatorname{dom}\left(\left(\mathcal{D}^{*}\right)_{\mathcal{P}^{\prime}}\right)$. Then this equality implies that $f$ is in the domain of the $L_{b}^{2}$ adjoint of $\mathcal{D}_{\mathcal{P}}$. The hard part is the other direction: Now assume that $f$ is in the domain of the $L_{b}^{2}$ adjoint of $\mathcal{D}_{\mathcal{P}}$; we need to show that $f \in \operatorname{dom}\left(\left(\mathcal{D}^{*}\right)_{\mathcal{P}^{\prime}}\right)$. By definition of the $L_{b}^{2}$ adjoint, we know that $f \in L_{b}^{2}(X, F)$ and $\mathcal{D}^{*} f \in L_{b}^{2}(X, E)$. Since $f \in L_{b}^{2}(X, F)$, the same proof as in the compact case shows that $\gamma_{0} f \in H_{b}^{-1 / 2}\left(H_{0}, F_{0}\right)$. In particular, $(\mathrm{Id}-\mathcal{P}) G_{0}^{*} \gamma_{0} f \in H_{b}^{-1 / 2}\left(H_{0}, E_{0}\right)$ defines a distribution acting on $H_{b}^{\infty}\left(H_{0}, E_{0}\right)$, so by the above Green's formula equality $(6.5)$, we see that this distribution must be zero. It remains to prove that $f \in H_{b}^{1}(X, F)$. 
To see this, we apply Lemma 6.4 to $\mathcal{D}^{*}$, to obtain an operator $Q^{\prime}$ such that for any $s>1 / 2, Q^{\prime}: H_{b}^{s}(X, E) \oplus H_{b}^{s+1 / 2}\left(H_{0}, F_{0}\right) \rightarrow H_{b}^{s+1}(X, F)$ is continuous and

$$
Q^{\prime} \circ\left(\begin{array}{c}
\mathcal{D}^{*} \\
\mathcal{P}^{\prime} \gamma_{0}
\end{array}\right)=\mathrm{Id}+\mathcal{S}^{\prime}: H_{b}^{s}(X, E) \rightarrow H_{b}^{s}(X, E),
$$

where $\mathcal{S}^{\prime}$ is a compact regularizing operator. We can apply this formula in the distributional sense to $f$, in which case, using that $\mathcal{D}^{*} f \in L_{b}^{2}(X, F)$ and, as a distribution, $\mathcal{P}^{\prime} \gamma_{0} f=0$, we see that

$$
f=Q^{\prime} \circ\left(\begin{array}{c}
\mathcal{D}^{*} f \\
0
\end{array}\right)-\mathcal{S}^{\prime} f \in H_{b}^{1}(X, F) .
$$

Our proof is now complete.

Finally, the relative index formula (1.8) follows directly from the following lemma.

Lemma 6.6. For $\mathcal{P}_{1}, \mathcal{P}_{2}, \mathcal{P}_{3} \in G r_{\infty}^{*}(\mathcal{D}), \mathcal{P}_{1} \mathcal{P}_{2}: \operatorname{ran} \mathcal{P}_{2} \rightarrow \operatorname{ran} \mathcal{P}_{1}$ is Fredholm. Its index $\operatorname{ind}\left(\mathcal{P}_{1}, \mathcal{P}_{2}\right):=\operatorname{ind}\left(\mathcal{P}_{1} \mathcal{P}_{2}: \operatorname{ran} \mathcal{P}_{2} \rightarrow \operatorname{ran} \mathcal{P}_{1}\right)$ satisfies

$$
\operatorname{ind}\left(\mathcal{P}_{1}, \mathcal{P}_{2}\right)=-\operatorname{ind}\left(\mathcal{P}_{2}, \mathcal{P}_{1}\right)=\operatorname{ind}\left(\operatorname{Id}-\mathcal{P}_{2}, \operatorname{Id}-\mathcal{P}_{1}\right)
$$

and the 'logarithm property'

$$
\operatorname{ind}\left(\mathcal{P}_{1}, \mathcal{P}_{3}\right)=\operatorname{ind}\left(\mathcal{P}_{1}, \mathcal{P}_{2}\right)+\operatorname{ind}\left(\mathcal{P}_{2}, \mathcal{P}_{3}\right)
$$

Proof. Since ind $\mathcal{P}_{1} \mathcal{P}_{2}=-\operatorname{ind}\left(\mathcal{P}_{1} \mathcal{P}_{2}\right)^{*}=\operatorname{ind} \mathcal{P}_{2} \mathcal{P}_{1}$, the equality $\operatorname{ind}\left(\mathcal{P}_{1}, \mathcal{P}_{2}\right)=$ $-\operatorname{ind}\left(\mathcal{P}_{2}, \mathcal{P}_{1}\right)$ is obvious. To prove that $\operatorname{ind}\left(\mathcal{P}_{2}, \mathcal{P}_{1}\right)=\operatorname{ind}\left(\operatorname{Id}-\mathcal{P}_{1}\right.$, Id $\left.-\mathcal{P}_{2}\right)$, we simply compute:

$$
\operatorname{ker} \mathcal{P}_{2} \mathcal{P}_{1}=\left\{\phi \mid\left(\mathrm{Id}-\mathcal{P}_{1}\right) \phi=0, \mathcal{P}_{2} \phi=0\right\}
$$

and

$$
\begin{aligned}
\operatorname{coker}\left[\mathcal{P}_{2} \mathcal{P}_{1}: \operatorname{ran}\left(\mathcal{P}_{1}\right) \rightarrow \operatorname{ran}\left(\mathcal{P}_{2}\right)\right] & \cong \operatorname{ker}\left[\left(\mathcal{P}_{2} \mathcal{P}_{1}\right)^{*}: \operatorname{ran}\left(\mathcal{P}_{2}\right) \rightarrow \operatorname{ran}\left(\mathcal{P}_{1}\right)\right] \\
& =\operatorname{ker}\left[\mathcal{P}_{1} \mathcal{P}_{2}: \operatorname{ran}\left(\mathcal{P}_{2}\right) \rightarrow \operatorname{ran}\left(\mathcal{P}_{1}\right)\right] \\
& =\left\{\phi \mid\left(\operatorname{Id}-\mathcal{P}_{2}\right) \phi=0, \mathcal{P}_{1} \phi=0\right\} .
\end{aligned}
$$

Replacing $\mathcal{P}_{2}$ with Id $-\mathcal{P}_{1}$ and $\mathcal{P}_{1}$ with $\mathrm{Id}-\mathcal{P}_{2}$ in (6.6) and (6.7), we obtain

$$
\operatorname{ker}\left(\operatorname{Id}-\mathcal{P}_{1}\right)\left(\operatorname{Id}-\mathcal{P}_{2}\right)=\left\{\phi \mid \mathcal{P}_{2} \phi=0,\left(\operatorname{Id}-\mathcal{P}_{1}\right) \phi=0\right\}
$$

and

$$
\operatorname{coker}\left(\mathrm{Id}-\mathcal{P}_{1}\right)\left(\mathrm{Id}-\mathcal{P}_{2}\right)=\left\{\phi \mid \mathcal{P}_{1} \phi=0,\left(\mathrm{Id}-\mathcal{P}_{2}\right) \phi=0\right\} .
$$

Comparing these spaces with $(6.6)$ and (6.7), we see that $\operatorname{ind}\left(\mathcal{P}_{2}, \mathcal{P}_{1}\right)=\operatorname{ind}(\mathrm{Id}-$ $\mathcal{P}_{1}$, Id $\left.-\mathcal{P}_{2}\right)$.

To prove the logarithm property, just note that by the logarithm property of the usual index, we have

$$
\operatorname{ind}\left(\mathcal{P}_{1}, \mathcal{P}_{2}\right)+\operatorname{ind}\left(\mathcal{P}_{2}, \mathcal{P}_{3}\right)=\operatorname{ind}\left[\mathcal{P}_{1} \mathcal{P}_{2} \mathcal{P}_{3}: \operatorname{ran}\left(\mathcal{P}_{3}\right) \rightarrow \operatorname{ran}\left(\mathcal{P}_{1}\right)\right]
$$

Hence, it remains to show that $\operatorname{ind}\left(\mathcal{P}_{1} \mathcal{P}_{3}\right)=\operatorname{ind}\left(\mathcal{P}_{1} \mathcal{P}_{2} \mathcal{P}_{3}\right)$. To see this, we write

$$
\mathcal{P}_{1} \mathcal{P}_{3}=\mathcal{P}_{1} \mathcal{P}_{2} \mathcal{P}_{3}+\mathcal{P}_{1}\left(\mathrm{Id}-\mathcal{P}_{2}\right) \mathcal{P}_{3}
$$

Since $\mathcal{P}_{1}=\mathcal{P}_{2}$ modulo compact, it follows that modulo compact, we have

$$
\mathcal{P}_{1}\left(\mathrm{Id}-\mathcal{P}_{2}\right) \mathcal{P}_{3}=\mathcal{P}_{2}\left(\mathrm{Id}-\mathcal{P}_{2}\right) \mathcal{P}_{3}=0 \text {. }
$$


Thus, $\mathcal{P}_{1} \mathcal{P}_{3}$ and $\mathcal{P}_{1} \mathcal{P}_{2} \mathcal{P}_{3}$ differ by a compact operator, so $\operatorname{ind}\left(\mathcal{P}_{1} \mathcal{P}_{3}\right)=\operatorname{ind}\left(\mathcal{P}_{1} \mathcal{P}_{2} \mathcal{P}_{3}\right)$, and our proof is complete.

6.2. Proof of Theorem 1.5. Let $M$ be a manifold with corners with an exact $b$-metric $g$ and let $\mathcal{D}: C^{\infty}(M, E) \rightarrow C^{\infty}(M, F)$ be a Dirac operator. Assume that $M$ is decomposed into two submanifolds $M_{ \pm}$with corners along a hypersurface $Y$ :

$$
M=M_{-} \cup M_{+} \quad, \quad \partial M_{-}=\partial M_{+}=Y
$$

where $\left.g\right|_{M_{ \pm}}$satisfies the conditions for manifold with multi-cylindrical end boundaries, in particular, $Y$ is admissible for both $M_{ \pm}$. Now let us prove Theorem 1.5: If

$$
\mathcal{D}: H_{b}^{1}(M, E) \rightarrow L_{b}^{2}(M, F)
$$

is Fredholm, then for arbitrary projections $\mathcal{P}_{ \pm} \in G r_{\infty}^{*}\left(\mathcal{D}_{ \pm}\right)$, we have

$$
\text { ind } \mathcal{D}=\operatorname{ind} \mathcal{D}_{\mathcal{P}_{-}}+\operatorname{ind} \mathcal{D}_{\mathcal{P}_{+}}-\operatorname{ind}\left(\mathcal{P}_{-}, \operatorname{Id}-\mathcal{P}_{+}\right)
$$

Before proceeding with the proof, we need a classical result about Fredholm pairs. Let $\mathcal{H}_{1}$ and $\mathcal{H}_{2}$ be closed subspaces in a Hilbert space $\mathcal{H}$. Then the pair $\left(\mathcal{H}_{1}, \mathcal{H}_{2}\right)$ is called as Fredholm pair of subspaces if

$$
\operatorname{dim}\left(\mathcal{H}_{1} \cap \mathcal{H}_{2}\right)<\infty \text { and } \operatorname{dim}\left(\mathcal{H}_{1}^{\perp} \cap \mathcal{H}_{2}^{\perp}\right)<\infty,
$$

in which case, we define the index of the pair $\left(\mathcal{H}_{1}, \mathcal{H}_{2}\right)$ to be the integer

$$
\operatorname{ind}\left(\mathcal{H}_{1}, \mathcal{H}_{2}\right):=\operatorname{dim}\left(\mathcal{H}_{1} \cap \mathcal{H}_{2}\right)-\operatorname{dim}\left(\mathcal{H}_{1}^{\perp} \cap \mathcal{H}_{2}^{\perp}\right)
$$

Now we recall the following classic result from [5, p. 263].

Lemma 6.7. Let $\mathcal{H}_{i}, i=1,2$, be closed subspaces in a Hilbert space $\mathcal{H}$ and let $P_{i}$ be the orthogonal projection onto $\mathcal{H}_{i}$. Then $\mathcal{H}_{1}$ and $\mathcal{H}_{2}$ form a Fredholm pair in $\mathcal{H}$ if and only if

$$
\left(\mathrm{Id}-P_{2}\right) P_{1}: \mathcal{H}_{1} \rightarrow \mathcal{H}_{2}^{\perp}
$$

is Fredholm, in which case,

$$
\operatorname{ind}\left(\mathcal{H}_{1}, \mathcal{H}_{2}\right)=\operatorname{ind}\left(\mathrm{Id}-P_{2}, P_{1}\right) .
$$

Proof. We simply compute:

$$
\operatorname{ker}\left(\mathrm{Id}-P_{2}\right) P_{1}=\left\{\phi \in \mathcal{H}_{1} \mid\left(\operatorname{Id}-P_{2}\right) \phi=0\right\}=\mathcal{H}_{1} \cap \mathcal{H}_{2}
$$

and

$$
\begin{aligned}
\operatorname{coker}\left[\left(\operatorname{Id}-P_{2}\right) P_{1}: \mathcal{H}_{1} \rightarrow \mathcal{H}_{2}^{\perp}\right] & \cong \operatorname{ker}\left[\left(\left(\operatorname{Id}-P_{2}\right) P_{1}\right)^{*}: \mathcal{H}_{2}^{\perp} \rightarrow \mathcal{H}_{1}\right] \\
& =\operatorname{ker}\left[P_{1}\left(\operatorname{Id}-P_{2}\right): \mathcal{H}_{2}^{\perp} \rightarrow \mathcal{H}_{1}\right] \\
& =\left\{\phi \in \mathcal{H}_{2}^{\perp} \mid P_{1} \phi=0\right\} \\
& =\mathcal{H}_{1}^{\perp} \cap \mathcal{H}_{2}^{\perp} .
\end{aligned}
$$

Now to prove the Bojarski conjecture let us first prove

$$
\text { ind } \mathcal{D}=\operatorname{ind}\left(\operatorname{Id}-\mathcal{C}_{-}, \mathcal{C}_{+}\right)=\operatorname{ind}\left(\operatorname{Id}-\mathcal{C}_{+}, \mathcal{C}_{-}\right) \text {. }
$$

By the unique continuation property [1, Cor. 1], it is obvious that

$$
\operatorname{ker} \mathcal{D} \cong \operatorname{ran}\left(\mathcal{C}_{-}\right) \cap \operatorname{ran}\left(\mathcal{C}_{+}\right) \quad, \quad \text { coker } \mathcal{D} \cong \operatorname{ker} \mathcal{D}^{*} \cong \operatorname{ran}\left(\mathcal{C}_{-}^{*}\right) \cap \operatorname{ran}\left(\mathcal{C}_{+}^{*}\right)
$$


where $\mathcal{C}_{ \pm}^{*}$ denotes the orthogonal Calderón projection for the restriction of $\mathcal{D}^{*}$ to $M_{ \pm}$. Applying Proposition 6.5, we obtain

$$
\begin{aligned}
\operatorname{ran}\left(\mathcal{C}_{-}^{*}\right) \cap \operatorname{ran}\left(\mathcal{C}_{+}^{*}\right) & =\operatorname{ran}\left(G_{0}\left(\mathrm{Id}-\mathcal{C}_{-}\right) G_{0}^{*}\right) \cap \operatorname{ran}\left(G_{0}\left(\mathrm{Id}-\mathcal{C}_{+}\right) G_{0}^{*}\right) \\
& \cong \operatorname{ran}\left(\operatorname{Id}-\mathcal{C}_{-}\right) \cap \operatorname{ran}\left(\operatorname{Id}-\mathcal{C}_{+}\right) \\
& \cong \operatorname{ran}\left(\mathcal{C}_{-}\right)^{\perp} \cap \operatorname{ran}\left(\mathcal{C}_{+}\right)^{\perp} .
\end{aligned}
$$

Thus, by Lemma 6.7,

$$
\text { ind } \begin{aligned}
\mathcal{D} & =\operatorname{dim} \operatorname{ker} \mathcal{D}-\operatorname{dim} \operatorname{coker} \mathcal{D} \\
& =\operatorname{dim}\left(\operatorname{ran}\left(\mathcal{C}_{-}\right) \cap \operatorname{ran}\left(\mathcal{C}_{+}\right)\right)-\operatorname{dim}\left(\operatorname{ran}\left(\mathcal{C}_{-}\right)^{\perp} \cap \operatorname{ran}\left(\mathcal{C}_{+}\right)^{\perp}\right) \\
& =\operatorname{ind}\left(\operatorname{Id}-\mathcal{C}_{-}, \mathcal{C}_{+}\right) .
\end{aligned}
$$

Finally, for arbitrary projections $\mathcal{P}_{ \pm} \in G r_{\infty}^{*}\left(\mathcal{D}_{ \pm}\right)$, we have

$$
\begin{aligned}
\operatorname{ind} \mathcal{D} & =\operatorname{ind}\left(\operatorname{Id}-\mathcal{C}_{-}, \mathcal{C}_{+}\right) \\
& =\operatorname{ind}\left(\operatorname{Id}-\mathcal{C}_{-}, \mathcal{P}_{+}\right)+\operatorname{ind}\left(\mathcal{P}_{+}, \mathcal{C}_{+}\right) \\
& =-\operatorname{ind}\left(\mathcal{C}_{-}, \operatorname{Id}-\mathcal{P}_{+}\right)+\operatorname{ind}\left(\mathcal{P}_{+}, \mathcal{C}_{+}\right) \\
& =-\operatorname{ind}\left(\mathcal{C}_{-}, \mathcal{P}_{-}\right)-\operatorname{ind}\left(\mathcal{P}_{-}, \operatorname{Id}-\mathcal{P}_{+}\right)+\operatorname{ind}\left(\mathcal{P}_{+}, \mathcal{C}_{+}\right) \\
& =\operatorname{ind}\left(\mathcal{P}_{-}, \mathcal{C}_{-}\right)+\operatorname{ind}\left(\mathcal{P}_{+}, \mathcal{C}_{+}\right)-\operatorname{ind}\left(\mathcal{P}_{-}, \operatorname{Id}-\mathcal{P}_{+}\right) \\
& =\operatorname{ind} \mathcal{D}_{\mathcal{P}_{-}}+\operatorname{ind} \mathcal{D}_{\mathcal{P}_{+}}-\operatorname{ind}\left(\mathcal{P}_{-}, \operatorname{Id}-\mathcal{P}_{+}\right) .
\end{aligned}
$$

\section{REFERENCES}

1. C. Bär: 'Zero sets of solutions to semilinear elliptic systems of first order', Invent. Math. 138, 183-202, 1999.

2. M. Sh. Birman and M. Z. Solomyak: 'On subspaces that admit a pseudodifferential projector', Vestnik Leningrad. Univ. Mat. Mekh. Astronom., no. vyp. 1, 18-25, 133, 1982.

3. D. Bleecker and B. Booß-Bavnbek: 'Spectral invariants of operators of Dirac type on partitioned manifolds', Aspects of Boundary Problems in Analysis and Geometry, Birkhäuser, Boston, 1130, 2004.

4. B. Bojarski: 'The abstract linear conjugation problem and Fredholm pairs of subspaces', In Memoriam I.N. Vekua, Tbilisi Univ, Tbilisi, 45-60, 1979 (Russian).

5. B. Booß-Bavnbek and K. P. Wojciechowski: 'Elliptic Boundary Problems for Dirac Operators', Birkhäuser, Boston, 1993.

6. L. Boutet de Monvel: 'Boundary problems for pseudo-differential operators' Acta Math. 126, no. 1-2, 11-51, 1971.

7. A. -P. Calderón: 'Boundary value problems for elliptic equations', Outlines Joint Sympos. Partial Differential Equations (Novosibirsk, 1963), Acad. Sci. USSR Siberian Branch, Moscow, pp. 303-304, 1963.

8. G. Grubb: 'Boundary problems for systems of partial differential operators of mixed order', $J$. Funct. Anal. 26, 131-165, 1977.

9. __ Functional calculus of pseudodifferential boundary problems, second ed., Progress in Math., Birkhäuser, Boston, 1996.

10. ___ 'Trace expansions for pseudodifferential boundary problems for Dirac-type operators and more general systems', Ark. Math. 37, 45-86, 1999.

11. G. Grubb and N. Kokholm: 'A global calculus of parameter-dependent pseudodifferential boundary problems in $L_{p}$ Sobolev spaces', Acta Math. 171, no. 2, 165-229, 1993.

12. L. Hörmander: 'Pseudo-differential operators and non-elliptic boundary problems', Ann. Math. 83, 129-209, 1966.

13. _ : The analysis of linear partial differential operators. III, second ed., Springer-Verlag, Berlin, 1985.

14. R. Lauter: 'On representations of $\psi^{*}$-algebras and $C^{*}$-algebras of $b$-pseudodifferential operators on manifolds with corners', J. Math. Sci. (New York) 98, no. 6, 684-705, 2000. 
15. R. Lauter and J. Seiler, Pseudodifferential analysis on manifolds with boundary-a comparison of b-calculus and cone algebra, Approaches to singular analysis (Berlin, 1999), Birkhäuser, Basel, 2001, pp. 131-166.

16. P. Loya: 'The structure of the resolvent of elliptic pseudodifferential operators', J. Funct. Anal. 184, no. 1, 77-135, 2001.

17. ___ 'Tempered operators and the heat kernel and complex powers of elliptic pseudodifferential operators', Comm. Partial Differential Equations 26, no. 7 \& 8, 1253-1321, 2001.

18. _ _ 'Dirac operators, Boundary Value Problems, and the b-Calculus', Contemp. Math. 366, 241-280, 2005.

19. P. Loya and R. Melrose: 'Fredholm perturbations of Dirac operators on manifolds with corners', Preprint, 2003.

20. P. Loya and J. Park: 'The spectral invariants and Krein's spectral shift function for Dirac operators on manifolds with multi-cylindrical end boundaries', Preprint, 2004.

21. _ G : 'Gluing formulæ for the spectral invariants of Dirac operators on noncompact manifolds', In preparation.

22. R. Mazzeo: 'Elliptic theory of differential edge operators. I', Comm. Partial Differential Equations 16, no. 10, 1615-1664, 1991.

23. R. B. Melrose: The Atiyah-Patodi-Singer Index Theorem, A.K. Peters, Wellesley, 1993.

24. _ Differential analysis on manifolds with corners, In preparation.

25. R.B. Melrose and V. Nistor: ' $K$-theory of $C^{*}$-algebras of $b$-pseudodifferential operators', Geom. Funct. Anal. 8, no. 1, 88-122, 1998.

26. R.B. Melrose and P. Piazza: 'Analytic K-theory on manifolds with corners', Adv. Math. 92, no. 1, 1-26, 1992 .

27. M. Mitrea and V. Nistor: 'A note on boundary value problems on manifolds with cylindrical ends', Aspects of Boundary Problems in Analysis and Geometry, Birkhäuser, Boston, 472-494, 2004.

28. E. Schrohe: 'Fréchet algebra techniques for boundary value problems on noncompact manifolds: Fredholm criteria and functional calculus via spectral invariance', Math. Nachr. 199, 145-185, 1999.

29. E. Schrohe and B.-W. Schulze: 'Boundary value problems in Boutet de Monvel's algebra for manifolds with conical singularities. II', Boundary value problems, Schrödinger operators, deformation quantization, Math. Top., vol. 8, Akademie Verlag, Berlin, 70-205, 1995.

30. R. T. Seeley: 'Singular integrals and boundary value problems', Amer. J. Math. 88, 781-809, 1966.

31. _ : 'Topics in pseudo-differential operators', Pseudo-Diff. Operators (C.I.M.E., Stresa, 1968), 167-305, 1969.

32. K.P. Wojciechowski: 'Elliptic operators and relative $K$-homology groups on manifolds with boundary', C. R. Math. Rep. Acad. Sci. Canada 7, no. 2, 149-154, 1985.

Department of Mathematics, Binghamton University, Vestal Parkway East, BingHAMTON, NY 13902, U.S.A.

E-mail address: paul@math.binghamton.edu

School of Mathematics, Korea Institute for Advanced Study, 207-43, Cheongnyangni 2-Dong, Dongdamun-gu, Seoul 130-722, Korea

E-mail address: jinsung@kias.re.kr 